\title{
¿Cómo abordar el déficit de información para la toma de decisiones a nivel local? Un análisis desde el diseño de un índice de desarrollo territorial sobre la base del registro administrativo
}

\author{
Ismael Toloza, Sergio Sánchez y Javier Carrasco ${ }^{1}$
}

\section{Resumen}

Este artículo aborda la problemática de la disponibilidad de información para la toma de decisiones a nivel subregional. Se propone construir un índice de desarrollo territorial (IDT) sobre la base de registros administrativos, que se compone de 19 variables, agrupadas en 6 dimensiones: i) capital cultural; ii) capital social; iii) capital humano; iv) capital institucional; v) capital físico, y vi) capital económico. El modelo propuesto se basa en el enfoque del desarrollo territorial y los factores estructurales que lo definen. La definición de las variables que componen el IDT se basa en la consulta a expertos y en análisis estadísticos multivariantes. Se intenta documentar gran parte de las fases, los procedimientos y las decisiones que dieron lugar al IDT, como una forma de abrir la discusión en torno a esta línea de investigación. Para finalizar se presenta el cálculo del IDT de los municipios chilenos de Angol y Carahue.

\section{Palabras clave}

Gobierno local, toma de decisiones, información, acceso a la información, desarrollo regional, indicadores del desarrollo, análisis multivariado, Chile

\section{Clasificación JEL}

018

Autores

Ismael Toloza Bravo es Doctor en Economía por la Universidad Autónoma de México e Investigador, Académico y Coordinador del Programa de Desarrollo Territorial y Competitividad del Instituto de Desarrollo Local y Regional (IDER) de la Universidad de la Frontera (Chile). Correo electrónico: ismael.toloza@ufrontera.cl.

Sergio Sánchez Cárcamo es Magister en Metodología de la Investigación Social por la Universidad Complutense de Madrid e Investigador del IDER de la Universidad de la Frontera (Chile). Correo electrónico: sergio.sanchez@ufrontera.cl.

Javier Carrasco Roa es Magíster en Estadística por la Universidad de Concepción (Chile) y Jefe de la Unidad de Infraestructura Estadística del Instituto Nacional de Estadísticas en La Araucanía (Chile). Correo electrónico: javier.carrasco@ine.cl. 


\section{Introducción}

En Chile no se dispone de información robusta para la toma de decisiones a nivel local ${ }^{2}$. Con esa afirmación comenzaba la sesión de la Comisión de Zonas Extremas, del Senado de Chile, de 6 de junio de 2016.

La comprensión del desarrollo a todo nivel (nacional, regional, territorial y local) ha transitado, desde enfoques vinculados casi exclusivamente al crecimiento económico, hacia modelos de interpretación multidimensional y holística. Entendido así el fenómeno, la posibilidad de desencadenar procesos de desarrollo está vinculada a consideraciones que permitan comprender los factores que definen estos procesos y las decisiones de gestión y política pública o privada que a la postre los afectan. Esta comprensión se hace más compleja a medida que el observador se adentra en la escala subnacional, toda vez que la disponibilidad de información y datos va disminuyendo debido a la escasa existencia de series robustas y periódicas, o a la no representatividad de las series existentes (por ejemplo, de la Encuesta de Caracterización Socioeconómica Nacional (CASEN) y la Nueva Encuesta Nacional de Empleo (NENE)). En ese contexto, la mayor parte de la información presentada en un formato que describa los indicadores de desarrollo y competitividad disponibles para la interpretación de fenómenos y la toma de decisiones a nivel local proviene de una combinación de fuentes. De ahí que la información secundaria disponible deba ser reforzada o complementada con el levantamiento de información primaria mediante encuestas o entrevistas. Este proceso entraña un encarecimiento de los medios de obtención de los indicadores, por lo que su reproducibilidad se ve limitada, o supeditada a la accesibilidad de recursos.

Esta escasa disponibilidad de información con respecto a la toma de decisiones a nivel territorial y local se ha transformado en una limitación y un desafío para el país. Esa situación se reconoció en el informe de la Comisión Asesora Presidencial en Descentralización y Desarrollo Regional (2014), donde se señala la necesidad de crear sistemas regionales integrados de información territorial para la toma de decisiones. En efecto, si bien a nivel local y territorial se dispone de muchos datos en los registros administrativos (municipales o sectoriales), es preciso determinar su disponibilidad y fiabilidad. También es necesario proceder a una reestructuración que permita aprovechar mejor los datos y proporcionar a los espacios subnacionales mayor y mejor información para la toma de decisiones de política pública y privada, de manera más pertinente a la complejidad y especificidad de cada territorio. La creciente demanda de estadísticas de nivel local y comunal ha sido una preocupación que ha abordado el Observatorio Social del Ministerio de Desarrollo Social (MIDESO), en conjunto con la oficina del Programa de las Naciones Unidas para el Desarrollo (PNUD) en Chile, desde 2011. En ese marco, se ha estudiado el desarrollo de una metodología que permita estimar áreas pequeñas a partir de los datos de la Encuesta CASEN de 2009 y que pueda reproducirse en versiones siguientes (Ministerio de Desarrollo Social, 2013).

En este contexto, el objeto del presente artículo es servir como contribución al menos en dos frentes. Por un lado, se intenta ayudar a subsanar las deficiencias de información a nivel local, sobre la base del diseño de un índice de desarrollo territorial (IDT) construido exclusivamente a partir de información de registro administrativo. Por otro, se releva el enfoque del desarrollo territorial desde el punto de vista de la identificación de los factores estructurales que determinan, como señala Boisier (2004), la emergencia sistémica del desarrollo.

\footnotetext{
2 Como señala Filgueira (2006), esta se entiende como la transformación de datos en indicadores y de estos en información que posibilite un mayor conocimiento e inteligencia social (pirámide evolutiva del proceso de incorporación de saberes técnicos a la política pública).
} 
Con esos fines, se conformó un marco teórico basado en el enfoque de los factores estructurales del desarrollo, que sirvió de base para el diseño del indicador. Se revisaron los indicadores subnacionales actualmente disponibles que aplican un enfoque territorial. Luego se procedió al diseño del IDT, sobre la base de la metodología para la construcción de índices compuestos, publicada en un manual titulado Handbook on Constructing Composite Indicators: Methodology and User Guide, de la OCDE (2008), y en la "Guía metodológica: diseño de indicadores compuestos de desarrollo sostenible", de Schuschny y Soto (2009). El indicador fue probado (es decir, calculado) en Carahue y Angol, dos comunas de la región de La Araucanía.

\section{Marco teórico}

\section{Desarrollo y dinámicas territoriales}

Como señala Alburquerque (2013), las antiguas políticas sectoriales que habían definido la administración central del Estado han ido dando paso a un enfoque territorial en sus diseños. Esto se debe a que los temas sustantivos del desarrollo (por ejemplo, las innovaciones productivas, los recursos humanos y la sostenibilidad ambiental) requieren un planteamiento desde los distintos ámbitos locales. En este contexto, Vázquez Barquero (2007) afirma que el desarrollo de un territorio consiste en un proceso de transformaciones y cambios endógenos, impulsados por la creatividad y la capacidad emprendedora existente en el territorio. Por lo tanto, los procesos de desarrollo no se pueden explicar solamente a partir de mecanismos externos. Estos procesos suelen producirse de forma endógena, es decir, al utilizar las capacidades del territorio mediante los mecanismos y fuerzas que caracterizan la acumulación de capitales y facilitan el progreso económico y social.

Con este particular enfoque del desarrollo se busca explicar cómo se mueven, expresan, interactúan y evolucionan las dinámicas en un espacio territorial determinado. Lo anterior es coherente con la afirmación del Consejo Económico y Social de Naciones Unidas (2011) en el sentido de que la sostenibilidad del bienestar depende de la capacidad de conseguir que los distintos capitales (natural, físico, humano, social, entre otros) sean fortalecidos y trasmitidos a las generaciones venideras.

En este contexto, y desde el enfoque de la teoría de la complejidad, Boisier (2004) entiende la estructura y la dinámica del desarrollo como el surgimiento de un sistema territorial complejo. Dentro de este es posible identificar subsistemas que, a su vez, están conformados por elementos más específicos. En el cuadro 1 se describen los seis subsistemas establecidos por Boisier.

Cuadro 1

Subsistemas según Boisier

\begin{tabular}{|c|c|}
\hline i) Subsistema axiológico & $\begin{array}{l}\text { Conformado por un conjunto de valores universales y singulares que definen la pertenencia a un territorio } \\
\text { y lo distinguen de otros territorios. }\end{array}$ \\
\hline ii) Subsistema de acumulación & $\begin{array}{l}\text { Incluye la acumulación de capital, de progreso técnico y de capital humano, factores de la actual teorización } \\
\text { del crecimiento endógeno. En la exogeneidad del desarrollo se puede constatar la importancia que tienen } \\
\text { estos factores en lo que se refiere al territorio, los proyectos políticos nacionales, la política económica global } \\
\text { y sectorial, y la demanda externa (en atención al mayor grado de apertura). Por ello se deben replantear las } \\
\text { formas de hacer política pública a nivel meso (regional), en tanto que la institucionalidad subnacional (región, } \\
\text { provincia, comuna) podría desempeñar un papel proactivo para hacer que tales decisiones sean más endógenas. }\end{array}$ \\
\hline iii) Subsistema decisional & $\begin{array}{l}\text { Configurado por agentes individuales, corporativos y colectivos, es la expresión humana del territorio. Por } \\
\text { tanto, no basta con la identificación y enumeración de cada agente, sino que interesa detectar con precisión el } \\
\text { proyecto que cada uno de ellos promueve, ya que en el momento de definir un proyecto político de desarrollo } \\
\text { (por ejemplo, un plan de desarrollo territorial) será necesario compatibilizar visiones no coincidentes. }\end{array}$ \\
\hline iv) Subsistema organizacional & $\begin{array}{l}\text { Compuesto por el universo de organizaciones públicas y privadas del territorio. Al igual que en la dimensión } \\
\text { anterior, no solo interesa definir el mapa organizacional y su densidad, sino los niveles de interrelación, } \\
\text { articulación y sinergia de sus acciones. }\end{array}$ \\
\hline
\end{tabular}


Cuadro 1 (conclusión)

v) Subsistema procedimental Se refiere a los procedimientos de la administración pública en el territorio, en atención a la prestación de servicios, el manejo del flujo de información y las acciones de apoyo al territorio para optimizar su posicionamiento en la globalización (oportunidades). En este contexto, se reconocen las características entrópicas (desordenadas) de la información pública, que elevan los costos de transacción y la incertidumbre, por lo que constituyen un escollo para el propio crecimiento económico. $\begin{array}{ll}\text { vi) Subsistema subliminal } & \text { Configurado por nueve categorías de capital intangible, a saber, el capital cognitivo, simbólico, } \\ \text { cultural, social, cívico, institucional, psicosocial, mediático y humano, que se consideran factores }\end{array}$ clave del desarrollo cuando este también se concibe como un resultado intangible.

Fuente: S. Boisier, "Una (re)visión heterodoxa del desarrollo (territorial): un imperativo categórico", Estudios Sociales, vol. 12, $N^{\circ} 23$, enero-junio de 2004 [en línea] https://dialnet.unirioja.es/descarga/articulo/2108278.pdf.

Entre estos seis subsistemas debe haber el mayor nivel posible de interconexión, que es a lo que se refieren los conceptos de "sinapsis neuronal" o "sincronía neuronal". Sin que existan sinapsis de alta densidad no es posible que surja desarrollo (Toloza, 2007).

Desde otro punto de vista, el Ministerio de Planificación Nacional y Política Económica (MIDEPLAN) de Costa Rica y el PNUD, en su guía metodológica "Marco conceptual, metodológico y operativo de la planificación regional” (González, Sánchez y Araúz, 2011), señalan que el enfoque de desarrollo territorial tiene en cuenta las dimensiones que se describen en el cuadro 2.

Cuadro 2

Dimensiones según el Programa de las Naciones Unidas para el Desarrollo

\begin{tabular}{ll}
\hline i) Dimensión económico-productiva & $\begin{array}{l}\text { Asociada a las decisiones y actividades que inciden en la capacidad de generación de riqueza con } \\
\text { un vínculo territorial a partir del conjunto de modalidades de sistemas productivos existentes y las } \\
\text { estructuras de distribución de la riqueza producida a nivel territorial. También se deben tener en } \\
\text { cuenta dimensiones como la inversión, la calidad y pertinencia de la formación del capital humano, } \\
\text { el capital social, la productividad, la generación de conocimientos e innovación y el conocimiento } \\
\text { popular, que se materializan en la competitividad y, sobre todo, en la inclusión y justicia económicas. }\end{array}$ \\
\hline Descrita como las aspiraciones y necesidades de la población perteneciente a un territorio, \\
atendiendo a su diversidad y condición socioeconómica, étnica, cultural y etaria. En esta dimensión \\
se debe garantizar la inclusión, la equidad, el respeto a la diversidad y la responsabilidad social.
\end{tabular}

Fuente: H. González, O. M. Sánchez e Y. Araúz, "Marco conceptual, metodológico y operativo de la planificación regional: guía metodológica para el diseño de la estrategia de abordaje regional en planificación del desarrollo", Ministerio de Planificación Nacional y Política Económica (MIDEPLAN)/Programa de las Naciones Unidas para el Desarrollo (PNUD), 2011 [en línea] https://documentos.mideplan.go.cr/alfresco/d/d/workspace/SpacesStore/8c656860-8f17-47df-a6e167cfccf89dff/CPR-001_1.pdf?guest=true.

En consecuencia, el desarrollo territorial se concibe a partir de un conjunto de elementos que permiten definirlo como un concepto teórico multideterminado y a la vez integral, que tiene en cuenta los aspectos económico-productivo, social, ambiental, cultural y político-institucional (González, Sánchez y Araúz, 2011).

Al respecto, Requejo (2007) desarrolla una perspectiva similar, a partir de un esquema basado en cinco tipos de capital: natural, físico-construido, humano, social y de imagen (véase el cuadro 3). 


\section{Cuadro 3}

Tipos de capital según Requejo

\begin{tabular}{ll}
\hline i) Capital natural & $\begin{array}{l}\text { Se entiende como la reserva de bienes que provienen del medio ambiente (suelos, bosques, agua, humedales, } \\
\text { entre otros), que proveen al territorio determinados bienes y servicios. }\end{array}$ \\
\hline ii) Capital físico-construido & $\begin{array}{l}\text { Comprende infraestructuras básicas, edificaciones residenciales, equipamiento público, instalaciones productivas } \\
\text { y de cualquier otro tipo, así como los equipos relacionados con la producción, los servicios públicos } \\
\text { y el consumo en un territorio. }\end{array}$ \\
\hline iii) Capital humano & $\begin{array}{l}\text { Representa la capacidad de la población de emprender procesos productivos y alcanzar mayores niveles de } \\
\text { bienestar y calidad de vida, mediante el uso de conocimientos, capacidades y aptitudes. }\end{array}$ \\
\hline iv) Capital social & $\begin{array}{l}\text { Se refiere a la capacidad de una sociedad de dar respuesta eficaz a sus necesidades y adaptarse, } \\
\text { mediante la asociación organizada. }\end{array}$ \\
\hline v) Capital de imagen & Puede entenderse como el reconocimiento del valor asociado a la identidad de un territorio. \\
\hline
\end{tabular}

Fuente: J. Requejo, "Clases de capital territorial. Clases de capital territorial y desarrollo sostenible”, 2007 [en línea] http://www. atclave.es/publicaciones/descargas/pub_desarrollo/17_clases_de_capital_territorial.pdf.

Del análisis de la primera parte de la guía metodológica del desarrollo económico territorial denominada Proposiciones para el proceso de construcción y realización de una estrategia de desarrollo económico territorial (Chile Emprende, 2005) se observa que los modelos descentralizados de gestión con enfoque territorial (comúnmente conocidos como modelos de desarrollo territorial) se estructuran a partir de una visión de desarrollo multidimensional que reconoce la existencia de diversos tipos de capital territorial. Estos se refieren al conjunto de recursos naturales, económicos, humanos, institucionales y culturales que otorgan especificidad e identidad a un territorio, condicionan su competitividad y representan su potencial endógeno de desarrollo. Al respecto, como toda forma de capital, no se trata de reservas fijas de recursos, pues parte de su esencia es su potencial de reproducirse y ampliarse.

El esquema teórico sobre el capital territorial propuesto por Chile Emprende (2005) hace referencia a cinco dimensiones (o tipos de capital): i) competitividad empresarial y laboral; ii) capital físico y acondicionamiento del territorio; iii) capital institucional; iv) capital social, y v) identidad y cultura. En el cuadro 4 se presenta una complementación y adaptación de estos conceptos en el marco del presente diseño.

\section{Cuadro 4}

Tipos de capital según Chile Emprende

\begin{tabular}{ll}
\hline i) Capital humano & $\begin{array}{l}\text { Referido a la masa crítica (recursos humanos), empresarial, laboral y académica, así como a las personas } \\
\text { con capacidad de liderazgo y su nivel de competitividad y desarrollo. }\end{array}$ \\
\hline ii) Capital financiero & $\begin{array}{l}\text { Referido a las inversiones, la disponibilidad de capital, oferta de financiamiento, instrumentos de intermediación, } \\
\text { capacidad de endeudamiento e institucionalidad financiera del territorio. }\end{array}$ \\
\hline iii) Capital físico y natural & $\begin{array}{l}\text { Asociado al acondicionamiento del territorio para proveer una infraestructura que permita aprovechar } \\
\text { las oportunidades de desarrollo, también se refiere al capital natural existente en el territorio y su vinculación } \\
\text { con dichas oportunidades. }\end{array}$ \\
\hline iv) Capital institucional & $\begin{array}{l}\text { Referido a la solidez de las instituciones del territorio, sean públicas o privadas, así como a las normas, leyes, } \\
\text { programas y plataformas de servicios, entre otras cosas. }\end{array}$ \\
\hline v) Capital social & $\begin{array}{l}\text { Vinculado, a efectos de este modelo teórico, al capital social comunitario y la existencia de redes, así como } \\
\text { de activos de cooperación y de gestión organizacional, de conflictos e historia de conflictos. }\end{array}$ \\
\hline vi) Capital simbólico & Asociado a la identidad y cultura de un territorio, conocimientos tácitos, saberes ancestrales, entre otras cosas. \\
\hline
\end{tabular}

Fuente: Chile Emprende, Guía metodológica del desarrollo territorial, Santiago, 2005 [en línea] http://www.dhl.hegoa.ehu.es/ ficheros/0000/0243/Chile_Estrategia_de_desarrollo_econ\%C3\%B3mico_territorial_2009.pdf.

En este contexto, y desde el punto de vista del desarrollo territorial, es necesario precisar con claridad las dimensiones del capital territorial que se deben poner en juego para captar las oportunidades y determinar el nivel de desarrollo relativo que exhiben en la actualidad dichas dimensiones. De esta manera se procura establecer las brechas y las variables que convendría dinamizar para aprovechar o captar las oportunidades de desarrollo de un territorio (Chile Emprende, 2005). 
En consecuencia, para poner en marcha procesos de desarrollo desde la perspectiva de los modelos descentralizados de gestión con enfoque territorial, es preciso emprender un proceso permanente de acumulación de estos capitales territoriales. El detonante de la emergencia sistémica consistiría en impulsar procesos (políticas, programas, proyectos y acciones) tendientes a aumentar los flujos de ingreso de capital territorial, y a su vez, disminuir los flujos de egreso. Se trata de producir una dinámica permanente de acumulación.

A efectos de esta investigación, esas últimas teorías basadas en los seis tipos de capital territorial, en tanto factores estructurales del desarrollo, son las que mayor sentido tienen en lo que se refiere a la tarea de abordar la construcción de un indicador de desarrollo territorial.

\section{Metodología}

La metodología utilizada se basó en el manual de apoyo para la construcción de índices compuestos, publicado por la OCDE en 2008, denominado Handbook on Constructing Composite Indicators: Methodology and User Guide y en la "Guía metodológica: diseño de indicadores compuestos de desarrollo sostenible", de Schuschny y Soto (2009).

En primer lugar, y partiendo de la definición de los tipos de capital territorial descritos en el marco teórico anterior, se procedió a definir las siguientes dimensiones del IDT: capital simbólico o cultural, capital humano, capital social, capital institucional, capital físico y de infraestructura y capital económico. Se determinó que las comunas piloto para la estimación del indicador serían las de Carahue y Angol ${ }^{3}$.

En una primera fase, la metodología de trabajo aplicada a la construcción del IDT se basó en talleres, plenarios y reuniones de trabajo periódicas, donde se fueron definiendo progresivamente cada una de las decisiones y los componentes que integran el modelo que se presenta.

El primer ejercicio consistió en formar un panel de expertos ${ }^{4}$. Su objetivo era ofrecer una primera aproximación respecto de las variables explicativas de cada dimensión. En este sentido, en el marco del desarrollo de un primer taller de trabajo, se procedió a establecer 107 variables iniciales, asociadas a las dimensiones señaladas. La identificación de las variables en esta etapa se basó principalmente en el criterio de cobertura temática, es decir, que las variables tuvieran una relación directa con la definición operativa de la dimensión.

\section{Primer ajuste del modelo}

Las 107 variables se sometieron a un filtro basado en el criterio de disponibilidad del dato en registro administrativo. De este modo se obtuvo un primer ajuste, de 107 a 88 variables (véase el anexo A1).

\section{Segundo ajuste del modelo}

De las 88 variables seleccionadas en el primer ajuste, se procedió a realizar un segundo ajuste del modelo basado en tres criterios adicionales: i) calidad de la información; ii) periodicidad,

\footnotetext{
${ }^{3}$ Esas comunas participan en el convenio de desempeño regional Universidad es Territorio (UNETE), organizado por la Universidad de La Frontera, lo que facilita la recolección de datos.

4 Constituido por Ismael Toloza, Doctor en Economía; Sergio Muñoz, Doctor en Estadística; Javier Carrasco, Magíster en Estadística; Alejandro Henríquez, Ingeniero Director de la oficina del Instituto Nacional de Estadísticas (INE) en La Araucanía; Sergio Sánchez, Magister en Metodologías de la Investigación; Camilo Rosas, sociólogo y profesor de Estadística Social; Ana María Alarcón, Doctora en Antropología, y Carlos Pineda, Paula Arias y Patricio Gallardo, profesionales de la unidad técnica del INE en La Araucanía.
} 
y iii) representatividad ${ }^{5}$. De esta forma, se descartó un total de 49 variables, lo que dio como resultado un modelo un tanto más acotado, con un total de 39 variables, como se muestra en el cuadro 5.

\section{Cuadro 5}

Segunda identificación de dimensiones y variables del índice de desarrollo territorial

\begin{tabular}{|c|c|c|}
\hline Dimensión & Identificación de la variable & Definición operacional \\
\hline \multirow[t]{5}{*}{ i) Capital cultural } & 1. Eventos culturales & $\begin{array}{l}\text { Número de eventos culturales } \\
\text { masivos al año, por comuna. }\end{array}$ \\
\hline & 2. Patrimonio cultural material & $\begin{array}{l}\text { Número de hitos patrimoniales declarados } \\
\text { por decreto, por comuna. }\end{array}$ \\
\hline & 3. Patrimonio cultural inmaterial & $\begin{array}{l}\text { Número de hitos patrimoniales inmateriales } \\
\text { registrados en el Sistema de Información para } \\
\text { la Gestión Patrimonial (SIGPA), por comuna. }\end{array}$ \\
\hline & 4. Comunidades y asociaciones indígenas & $\begin{array}{l}\text { Número de comunidades y asociaciones } \\
\text { indígenas, por comuna. }\end{array}$ \\
\hline & $\begin{array}{l}\text { 5. Marcas territoriales y } \\
\text { denominaciones de origen }\end{array}$ & $\begin{array}{l}\text { Número de indicaciones geográficas y } \\
\text { denominaciones de origen, por comuna. }\end{array}$ \\
\hline ii) Capital social & 6. Agrupaciones con fines sociales & $\begin{array}{l}\text { Número de agrupaciones con fines sociales y con } \\
\text { personalidad jurídica vigente, por comuna. En este } \\
\text { caso se incluyen los centros de madres, de padres } \\
\text { y de apoderados, los centros u organizaciones de } \\
\text { adultos mayores, los clubes deportivos, las juntas } \\
\text { de vecinos y otras agrupaciones funcionales. }\end{array}$ \\
\hline \multirow[t]{4}{*}{ iii) Capital humano } & 7. Años de escolaridad & Años de escolaridad media, por comuna. \\
\hline & 8. Pruebas de selección universitaria (PSU) & Promedio obtenido en las PSU, por comuna. \\
\hline & 9. Población económicamente activa (PEA) & $\begin{array}{l}\text { Porcentaje de población en edad de trabajar } \\
\text { (ocupados y desocupados), por comuna. }\end{array}$ \\
\hline & 10. Empleabilidad & $\begin{array}{l}\text { Porcentaje de la PEA que se encuentra } \\
\text { ocupada, por comuna. }\end{array}$ \\
\hline \multirow[t]{6}{*}{ iv) Capital institucional } & 11. Participación del FCM & $\begin{array}{l}\text { Porcentaje del ingreso total municipal } \\
\text { proveniente del FCM, por municipio. }\end{array}$ \\
\hline & 12. Ingresos municipales & $\begin{array}{l}\text { Ingresos municipales percibidos en millones } \\
\text { de pesos chilenos, por municipio. }\end{array}$ \\
\hline & 13. Gastos municipales & $\begin{array}{l}\text { Gastos municipales devengados per cápita en } \\
\text { millones de pesos chilenos, por municipio. }\end{array}$ \\
\hline & 14. Plan de Desarrollo Comunal (PLADECO) & Existencia de PLADECO vigente, por municipio. \\
\hline & $\begin{array}{l}\text { 15. Plan Anual de Educación } \\
\text { Municipal (PADEM) }\end{array}$ & Existencia de PADEM vigente, por municipio. \\
\hline & 16. Plan Anual de Salud Municipal (PASAM) & Existencia de PASAM vigente, por municipio. \\
\hline \multirow[t]{13}{*}{ v) Capital físico y de infraestructura } & 17. Caminos de hormigón & Kilómetros de caminos de hormigón, por comuna. \\
\hline & 18. Caminos asfaltados & Kilómetros de caminos de asfalto, por comuna. \\
\hline & 19. Caminos de ripio & Kilómetros de caminos de ripio, por comuna. \\
\hline & 20. Cobertura telefónica & Porcentaje de cobertura de telefonía fija, por comuna. \\
\hline & 21. Cobertura de Internet & $\begin{array}{l}\text { Porcentaje de cobertura de Internet } \\
\text { (red fija), por comuna. }\end{array}$ \\
\hline & 22. Metros cuadrados construidos & $\begin{array}{l}\text { Metros cuadrados construidos per cápita con } \\
\text { recepción definitiva durante el año, por comuna. }\end{array}$ \\
\hline & 23. Cobertura de agua potable & Porcentaje de cobertura de agua potable, por comuna. \\
\hline & 24. Cobertura de alcantarillado & Porcentaje de cobertura de alcantarillado, por comuna. \\
\hline & 25. Cobertura de la red eléctrica & Porcentaje de cobertura de la red eléctrica, por comuna. \\
\hline & 26. Establecimientos educacionales & Número de establecimientos educacionales, por comuna. \\
\hline & 27. Establecimientos de salud & Número de establecimientos de salud, por comuna. \\
\hline & 28. Áreas silvestres protegidas por el Estado & $\begin{array}{l}\text { Kilómetros cuadrados de áreas silvestres } \\
\text { protegidas, por comuna. }\end{array}$ \\
\hline & 29. Bosques nativos & Kilómetros cuadrados de bosque nativo, por comuna. \\
\hline
\end{tabular}

5 Es decir, calidad de la información, referida a la existencia de una metodología validada para la generación y registro del dato; periodicidad, asociada a la fecha y la frecuencia de actualización del dato, y representatividad, entendida como la validez estadística del dato a nivel comunal. 
Cuadro 5 (conclusión)

\begin{tabular}{|c|c|c|}
\hline $\begin{array}{l}\text { Dimensión } \\
\text { vi) Capital económico }\end{array}$ & Identificación de la variable & Definición operacional \\
\hline \multirow[t]{9}{*}{ vi) Capital económico } & 30. Microempresas & Número de microempresas, por comuna. \\
\hline & 31. Trabajadores de microempresas & $\begin{array}{l}\text { Número de trabajadores de } \\
\text { microempresas, por comuna. }\end{array}$ \\
\hline & 32. Pequeñas empresas & Número de pequeñas empresas, por comuna. \\
\hline & 33. Trabajadores de pequeñas empresas & $\begin{array}{l}\text { Número de trabajadores de pequeñas } \\
\text { empresas, por comuna. }\end{array}$ \\
\hline & 34. Medianas empresas & Número de medianas empresas, por comuna. \\
\hline & 35. Trabajadores de medianas empresas & $\begin{array}{l}\text { Número de trabajadores de medianas } \\
\text { empresas, por comuna. }\end{array}$ \\
\hline & 36. Grandes empresas & Número de grandes empresas, por comuna. \\
\hline & 37. Trabajadores de grandes empresas & $\begin{array}{l}\text { Número de trabajadores de grandes } \\
\text { empresas, por comuna. }\end{array}$ \\
\hline & 38. Inversiones & Número de proyectos en ejecución, por comuna. \\
\hline vii) Otras variables & 39. Pobreza & Porcentaje de incidencia de pobreza, por comuna. \\
\hline
\end{tabular}

Fuente: Elaboración propia.

En correspondencia con lo expuesto anteriormente, en el documento "Indicadores sociales y marcos conceptuales para la medición social", de Cerda y Vera (2008), se describe una serie de aspectos que vale la pena tener en cuenta a la hora de identificar variables para el diseño de índices. Entre estos cabe mencionar la existencia de datos, la cobertura de zonas geográficas, la accesibilidad a los datos y su comparabilidad.

\section{a) Análisis de coherencia}

Era necesario contar con datos analizables para probar la estructura y el funcionamiento del modelo, teniendo en cuenta que el acceso a datos de nivel comunal es una tarea compleja. Por ese motivo, y con fines analíticos, se consideró que la opción más práctica sería someter el modelo a una serie de análisis multivariantes, mediante el uso de datos a nivel regional, dada la mayor disponibilidad de información respecto de las variables analizadas a ese nivel (véase el anexo A2). Según Hair, Anderson y Tatham (1999), una de las ventajas de las técnicas multivariantes radica en la posibilidad de realizar de forma simultánea el análisis de un conjunto de variables sobre un objeto, lo que produce resultados que no sería posible obtener con métodos univariantes.

Dicho análisis persigue distintos propósitos. En primer lugar, se busca constatar la validez del modelo, es decir, que el conjunto de variables seleccionadas, en definitiva, mida un objeto asociado al desarrollo territorial. Con ese fin se realizaron análisis de correlación y regresión (Cuadras, 2007) del indicador construido, respecto a indicadores de pobreza y desarrollo. En segundo lugar, se efectuaron análisis de conglomerados (Figueras, 2001) con la idea de entender la forma en que se agrupaban los datos del conjunto de las variables en relación con los casos (en este ejercicio, las regiones). En tercer lugar, se realizaron análisis factoriales de componentes principales (García, Gil y Rodríguez, 2001) respecto de cada dimensión, con el objeto de analizar la estructura interna de las variables y la forma en que estas se agrupan en determinados factores. El análisis factorial con miras a la reducción de dimensiones es importante en la medida en que permite evaluar, con base en determinadas pruebas estadísticas, las variables más indicadas a la hora de explicar el comportamiento o la variación de un determinado objeto y las que podrían quedar fuera del modelo. De este modo se busca reducir la dimensionalidad del conjunto de variables iniciales, tratando de conservar la mayor parte de la información proporcionada por las variables observadas (Cea d'Ancona, 2002).

En el análisis multivariante de los datos se utilizó principalmente el paquete estadístico SPSS y R-project. Este software (a diferencia de otros, más orientados al análisis descriptivo) ofrece una serie de métodos avanzados de modelación estadística y análisis multivariado. 


\section{b) Análisis multivariante}

Con el fin de estudiar la factibilidad de resumir la información y reducir la dimensionalidad del polinomio en cuestión, se procedió en primera instancia a evaluar la pertinencia de realizar un análisis factorial (AF) con extracción, por medio del método de análisis de componentes principales (ACP) y con rotación ortogonal basada en el método Varimax. El análisis factorial permite encontrar combinaciones de variables a partir de las interrelaciones subyacentes entre estas. Tales combinaciones, llamadas factores, tienen la finalidad de representar de forma condensada la información contenida en las variables. Esto se logra al utilizar una cantidad menor de factores que reúnen gran parte de la información, lo que se aprecia en el porcentaje de varianza acumulada por los factores. A su vez, el método de componentes principales permite conformar factores que reúnan una gran variabilidad, de modo que se reduce la dimensión de los datos. El método de rotación Varimax modifica la representación de los factores, una vez construidos, para destacar la participación de las variables que los componen y facilitar su interpretabilidad.

En cuanto a la evaluación de la pertinencia de realizar un análisis factorial, es necesario comprobar, en cada una de las dimensiones del IDT, que los datos posean ciertas características que hagan idóneo su uso. Para ello se examinó la matriz de correlaciones y se determinó así el grado de interrelación entre las variables objeto de estudio. A su vez, se aplicó a cada dimensión la prueba de esfericidad de Bartlett, que indica si las correlaciones observadas resultan significativas. Se calculó además el coeficiente Kaiser-Meyer-Olkin (KMO), que indica el grado en que la información contenida en esas variables puede explicarse a partir de las demás. Ello resulta idóneo para el análisis factorial.

Luego se procedió a estudiar las comunalidades, que ponen de relieve hasta qué punto la varianza de una variable queda explicada por la solución factorial. También se determinaron las cargas de las variables en los factores rotados. A continuación se explica con detalle el análisis realizado de cada una de las dimensiones.

\section{i. Dimensión cultural}

La dimensión cultural está representada por las variables siguientes: eventos culturales (DC1), patrimonio cultural inmaterial (DC2), patrimonio cultural material (DC3), marcas territoriales (DC4) y comunidades indígenas (DC5) (véase el cuadro 6).

Cuadro 6

Matriz de correlaciones de la dimensión cultural

\begin{tabular}{|c|c|c|c|c|c|}
\hline Variables & DC1 & DC2 & DC3 & DC4 & DC5 \\
\hline $\mathrm{DC1}$ & 1,000 & & & & \\
\hline DC2 & 0,420 & 1,000 & & & \\
\hline DC3 & $0,967^{\star \star \star}$ & $0,454^{* *}$ & 1,000 & & \\
\hline DC4 & 0,398 & 0,400 & $0,456^{\star \star}$ & 1,000 & \\
\hline DC5 & $-0,078$ & 0,303 & $-0,018$ & 0,037 & 1,000 \\
\hline
\end{tabular}

Fuente: Elaboración propia.

Nota: $\quad$ *Valor $p<0,01 ;{ }^{\star *}$ valor $p<0,05 ;{ }^{* \star *}$ valor $p<0,001$

En el cuadro 6 se muestran las correlaciones entre las variables de la dimensión cultural. Se aprecia un alto grado de relación entre ellas. Así, por ejemplo, se observa que la correlación de Pearson entre eventos culturales y patrimonio cultural material es estadísticamente significativa (valor $p<0,001$ ). Lo mismo sucede con las correlaciones existentes entre los pares de variables de patrimonio cultural inmaterial y patrimonio cultural material, por una parte, y patrimonio cultural inmaterial y marcas territoriales, por otra (valor $\mathrm{p}<0,05)$. 
A su vez, la prueba de esfericidad de Bartlett resultó ser significativa (sig. < 0,001), lo que indica la existencia de correlaciones importantes, de lo que se infiere la pertinencia de continuar con el análisis. Por otro lado, el coeficiente KMO interno de la dimensión cultural es cercano a 0,6, por lo que no se desaconseja realizar el AF (véase el cuadro 7).

Cuadro 7

Prueba de esfericidad de Bartlett y coeficiente KMO (dimensión cultural)

\begin{tabular}{lcc}
\hline Medida de adecuación muestral de Kaiser-Meyer-Olkin & & 0,598 \\
\hline Prueba de esfericidad de Bartlett & Chi-cuadrado aproximado & 40,149 \\
\cline { 2 - 3 } & $\mathrm{gl}$ & 10 \\
\cline { 2 - 3 } & $\mathrm{Sig}$. & 0,000 \\
\hline
\end{tabular}

Fuente: Elaboración propia.

Con todos estos análisis, se concluye que es apropiado aplicar el análisis factorial a esta serie de datos. Así pues, se procedió a seleccionar tres factores, que en conjunto explican un 89,714\% de la varianza total. El primer factor acumula un $51,960 \%$ de la varianza total y el segundo, un $23,757 \%$. Entretanto, el tercer factor explica un 13,997\% (véase el cuadro 8).

\section{Cuadro 8}

Varianza explicada (dimensión cultural)

\begin{tabular}{lccccccc}
\hline \multirow{2}{*}{ Componente } & \multicolumn{3}{c}{ Autovalores iniciales } & & \multicolumn{2}{c}{ Suma de las saturaciones al cuadrado de la rotación } \\
\cline { 2 - 3 } & Total & $\begin{array}{c}\text { Porcentaje de } \\
\text { la varianza }\end{array}$ & $\begin{array}{c}\text { Porcentaje } \\
\text { acumulado }\end{array}$ & & Total & $\begin{array}{c}\text { Porcentaje de } \\
\text { la varianza }\end{array}$ & $\begin{array}{c}\text { Porcentaje } \\
\text { acumulado }\end{array}$ \\
\hline 1 & 2,598 & 51,960 & 51,960 & & 2,598 & 51,960 & 51,960 \\
\hline 2 & 1,188 & 23,757 & 75,717 & & 1,188 & 23,757 & 75,717 \\
\hline 3 & 0,700 & 13,997 & 89,714 & & 0,700 & 13,997 & 89,714 \\
\hline 5 & 0,485 & 9,705 & 99,419 & & & & \\
\hline
\end{tabular}

Fuente: Elaboración propia.

Por último, al analizar la matriz de componentes rotados, se aprecia que las variables de eventos culturales y patrimonio cultural material se van modificando en la misma dirección y se agrupan en el primer componente (algunas podrían quedar fuera del polinomio). A su vez, la variable del patrimonio cultural inmaterial no se agrupa en ningún componente, lo que significa que proporciona información que no se puede resumir en ninguna combinación lineal de las demás variables. Cabe subrayar además el valor de las comunalidades, que fueron superiores al 69,0\% (véase el cuadro 9).

Cuadro 9

Matriz de componentes rotados y comunalidades (dimensión cultural)

\begin{tabular}{lcccc}
\hline \multicolumn{2}{l}{ Matriz de componentes rotados } & \multicolumn{2}{c}{ Componente } & \multirow{2}{*}{ Comunalidades } \\
\cline { 2 - 4 } Variables & 1 & 2 & 3 & \\
\hline DC1 & 0,973 & $-0,015$ & 0,173 & 0,977 \\
\hline DC2 & 0,415 & 0,572 & 0,443 & 0,695 \\
\hline DC3 & 0,956 & 0,040 & 0,232 & 0,969 \\
\hline DC4 & 0,218 & 0,020 & 0,950 & 0,950 \\
\hline DC5 & $-0,091$ & 0,940 & $-0,037$ & 0,892 \\
\hline
\end{tabular}

Fuente: Elaboración propia. 


\section{ii. Dimensión del capital humano}

La dimensión del capital humano está constituida en esta etapa por las variables de años de escolaridad (DE1), puntaje medio obtenido en las pruebas de selección universitaria (PSU) (DE2), tasa de participación en la fuerza de trabajo (DE3), tasa de ocupación (DE4), ingresos regionales (DE5) y gastos regionales (DE6).

Respecto a las correlaciones, se observa una marcada relación entre la tasa de participación en la fuerza de trabajo y la tasa de ocupación, así como una correlación entre los ingresos y los gastos regionales (valor $p<0,01$ ) (véase el cuadro 10).

Cuadro 10

Matriz de correlaciones de la dimensión del capital humano

\begin{tabular}{|c|c|c|c|c|c|c|}
\hline Variables & $\mathrm{DE} 1$ & DE2 & DE3 & DE4 & DE5 & DE6 \\
\hline DE1 & 1,000 & & & & & \\
\hline DE2 & 0,058 & 1,000 & & & & \\
\hline DE3 & $0,552^{\star \star}$ & 0,316 & 1,000 & & & \\
\hline DE4 & $0,557^{\star \star}$ & 0,302 & $0,915^{\star \star \star}$ & 1,000 & & \\
\hline DE5 & 0,382 & $0,747^{\star \star *}$ & 0,290 & 0,299 & 1,000 & \\
\hline DE6 & 0,383 & $0,750^{\text {*** }}$ & 0,298 & 0,307 & $0,999^{* \star *}$ & 1,000 \\
\hline
\end{tabular}

Fuente: Elaboración propia.

Nota: $\quad$ *Valor $p<0,01 ;{ }^{* *}$ valor $p<0,05 ;{ }^{* *}$ valor $p<0,001$

De igual forma, la prueba de esfericidad de Bartlett resultó ser significativa (sig. < 0,001), mientras que el índice KMO interno de la dimensión fue de 0,673, por lo que es pertinente realizar el AF (véase el cuadro 11).

Cuadro 11

Prueba de esfericidad de Bartlett y KMO (dimensión del capital humano)

\begin{tabular}{lcr}
\hline Medida de adecuación muestral de Kaiser-Meyer-Olkin & & 0,673 \\
\hline Prueba de esfericidad de Bartlett & Chi-cuadrado aproximado & 127,581 \\
\cline { 2 - 3 } & $\mathrm{gl}$ & 15 \\
\cline { 2 - 3 } & $\mathrm{Sig}$ & 0,000 \\
\hline
\end{tabular}

Fuente: Elaboración propia.

Por otro lado, en esta dimensión se seleccionaron tres factores que explican el 95,602\% de la variabilidad total de la dimensión. El primero acumula un 56,922\% de la varianza total, mientras que el segundo explica un $27,080 \%$ de la variabilidad de la dimensión. Por su parte, el tercer factor reúne un $11,601 \%$ de la varianza (véase el cuadro 12).

Cuadro 12

Varianza explicada (capital humano)

\begin{tabular}{|c|c|c|c|c|c|c|}
\hline \multirow[b]{2}{*}{ Componente } & \multicolumn{3}{|c|}{ Autovalores iniciales } & \multicolumn{3}{|c|}{ Suma de las saturaciones al cuadrado de la rotación } \\
\hline & Total & $\begin{array}{l}\text { Porcentaje de } \\
\text { la varianza }\end{array}$ & $\begin{array}{l}\text { Porcentaje } \\
\text { acumulado }\end{array}$ & Total & $\begin{array}{l}\text { Porcentaje de } \\
\text { la varianza }\end{array}$ & $\begin{array}{l}\text { Porcentaje } \\
\text { acumulado }\end{array}$ \\
\hline 1 & 3,415 & 56,922 & 56,922 & 3,415 & 56,922 & 56,922 \\
\hline 2 & 1,625 & 27,080 & 84,002 & 1,625 & 27,080 & 84,002 \\
\hline 3 & 0,696 & 11,601 & 95,602 & 0,696 & 11,601 & 95,602 \\
\hline 4 & 0,180 & 2,993 & 98,595 & & & \\
\hline 5 & 0,084 & 1,402 & 99,997 & & & \\
\hline 6 & 0,000 & 0,003 & 100,000 & & & \\
\hline
\end{tabular}

Fuente: Elaboración propia. 
Los resultados de la rotación muestran que las variables de puntaje medio en las PSU, los ingresos regionales y los gastos regionales han quedado agrupadas en el primer factor. Entretanto, la tasa de participación y la tasa de ocupación se agrupan en el segundo. Por lo demás, el tercer factor se compone principalmente de los años de escolaridad (véase el cuadro 13).

Cuadro 13

Matriz de componentes rotados y comunalidades (dimensión del capital humano)

\begin{tabular}{lcccc}
\hline \multirow{2}{*}{ Variables } & \multicolumn{3}{c}{ Componente } & \multirow{2}{*}{ Comunalidades } \\
\cline { 2 - 4 } & 1 & 2 & 3 & 0,958 \\
\hline DE1 & 0,149 & 0,385 & 0,887 & 0,912 \\
\hline DE2 & 0,878 & 0,267 & $-0,265$ & 0,955 \\
\hline DE3 & 0,151 & 0,945 & 0,198 & 0,949 \\
\hline DE4 & 0,152 & 0,938 & 0,215 & 0,979 \\
\hline DE5 & 0,950 & 0,091 & 0,263 & 0,979 \\
\hline DE6 & 0,950 & 0,100 & 0,260 & \\
\hline
\end{tabular}

Fuente: Elaboración propia.

A su vez, las comunalidades de todas las variables fueron superiores al 90,0\%.

De esta forma, los resultados indican que se puede reducir la dimensionalidad del capital educacional, dada la alta correlación entre las variables y el sentido de su variabilidad.

\section{iii. Dimensión física}

La dimensión física está constituida inicialmente por las siguientes variables: caminos de hormigón (DF1), caminos asfaltados (DF2), caminos de ripio (DF3), caminos de tierra (DF4), cobertura telefónica (DF5), cobertura de Internet (DF6), cobertura de agua potable (DF7), cobertura de alcantarillado (DF8), cobertura de la red eléctrica (DF9), establecimientos educacionales (DF10), establecimientos de salud (DF11) y metros cuadrados construidos al año más servicios (DF12) (véase el cuadro 14).

Cuadro 14

Matriz de correlaciones de la dimensión física

\begin{tabular}{|c|c|c|c|c|c|c|c|c|c|c|c|c|}
\hline Variables & DF1 & DF2 & DF3 & DF4 & DF5 & DF6 & DF7 & DF8 & DF9 & DF10 & DF11 & DF12 \\
\hline DF1 & 1,000 & & & & & & & & & & & \\
\hline DF2 & $-0,337$ & 1,000 & & & & & & & & & & \\
\hline DF3 & 0,350 & 0,318 & 1,000 & & & & & & & & & \\
\hline DF4 & $-0,442^{\star \star}$ & $0,527^{* *}$ & 0,209 & 1,000 & & & & & & & & \\
\hline DF5 & 0,123 & 0,159 & $-0,188$ & $-0,291$ & 1,000 & & & & & & & \\
\hline DF6 & 0,120 & 0,196 & $-0,174$ & $-0,287$ & $0,998^{* \star *}$ & 1,000 & & & & & & \\
\hline DF7 & 0,098 & $-0,105$ & $-0,499^{* *}$ & $-0,298$ & 0,191 & 0,200 & 1,000 & & & & & \\
\hline DF8 & $-0,128$ & $-0,256$ & $-0,620^{* \star}$ & 0,049 & 0,244 & 0,241 & $0,591^{\star *}$ & 1,000 & & & & \\
\hline DF9 & $-0,664^{\star *}$ & 0,176 & $-0,308$ & $0,549^{\star \star}$ & $-0,338$ & $-0,346$ & 0,154 & 0,220 & 1,000 & & & \\
\hline DF10 & 0,169 & 0,441 & 0,185 & $-0,227$ & $0,877^{\star \star \star}$ & $0,894^{\star \star \star}$ & $-0,053$ & $-0,130$ & $-0,426$ & 1,000 & & \\
\hline DF11 & 0,191 & $0,543^{* *}$ & 0,350 & $-0,127$ & $0,788^{\star \star \star}$ & $0,808^{\star \star \star}$ & $-0,116$ & $-0,244$ & $-0,419$ & $0,977^{\star \star \star}$ & 1,000 & \\
\hline DF12 & 0,126 & 0,151 & $-0,143$ & $-0,273$ & $0,995^{\text {***}}$ & $0,991^{\star \star \star}$ & 0,099 & 0,196 & $-0,365$ & $0,888^{\star \star \star}$ & $0,800^{\star \star \star}$ & 1,000 \\
\hline
\end{tabular}

Fuente: Elaboración propia.

Nota: $\quad$ *Valor $p<0,01 ;{ }^{\star \star}$ valor $p<0,05 ;{ }^{\star \star *}$ valor $p<0,001$

La matriz de correlaciones muestra un alto grado de relación entre las variables. Cabe destacar, por ejemplo, la correlación estadísticamente significativa (valor $\mathrm{p}<0,001$ ) que existe entre la cobertura telefónica y la de Internet, o la cobertura de agua potable y la de alcantarillado (véase el cuadro 14).

Por ese mismo motivo, la prueba de esfericidad de Bartlett es estadísticamente significativa (sig. < 0,001). Sin embargo, se aprecia que el índice KMO, de 0,438, es bajo (véase el cuadro 15). 


\section{Cuadro 15}

Prueba de esfericidad de Bartlett y coeficiente KMO (dimensión física)

\begin{tabular}{lcr}
\hline Medida de adecuación muestral de Kaiser-Meyer-Olkin & & 0,438 \\
\hline Prueba de esfericidad de Bartlett & Chi-cuadrado aproximado & 260,302 \\
\cline { 2 - 3 } & $\mathrm{gl}$ & 66 \\
\cline { 2 - 3 } & Sig. & 0,000 \\
\hline
\end{tabular}

Fuente: Elaboración propia.

En esta dimensión se han seleccionado cuatro factores que, en conjunto, explican un 89,919\% de la varianza total de la dimensión. A continuación se muestran en detalle los valores propios asociados a cada uno de esos factores, así como el porcentaje de varianza que existe entre ellos (véase el cuadro 16).

Cuadro 16

Varianza explicada (dimensión física)

\begin{tabular}{|c|c|c|c|c|c|c|}
\hline \multirow[b]{2}{*}{ Componente } & \multicolumn{3}{|c|}{ Autovalores iniciales } & \multicolumn{3}{|c|}{ Suma de las saturaciones al cuadrado de la rotación } \\
\hline & Total & $\begin{array}{l}\text { Porcentaje de } \\
\text { la varianza }\end{array}$ & $\begin{array}{l}\text { Porcentaje } \\
\text { acumulado }\end{array}$ & Total & $\begin{array}{l}\text { Porcentaje de } \\
\text { la varianza }\end{array}$ & $\begin{array}{l}\text { Porcentaje } \\
\text { acumulado }\end{array}$ \\
\hline 1 & 5,040 & 42,001 & 42,001 & 5,040 & 42,001 & 42,001 \\
\hline 2 & 2,697 & 22,472 & 64,473 & 2,697 & 22,472 & 64,473 \\
\hline 3 & 2,259 & 18,823 & 83,295 & 2,259 & 18,823 & 83,295 \\
\hline 4 & 0,795 & 6,623 & 89,919 & 0,795 & 6,623 & 89,919 \\
\hline 5 & 0,508 & 4,615 & 95,460 & & & \\
\hline 6 & 0,294 & 2,674 & 98,134 & & & \\
\hline 7 & 0,149 & 1,352 & 99,486 & & & \\
\hline 8 & 0,036 & 0,331 & 99,817 & & & \\
\hline 9 & 0,018 & 0,165 & 99,982 & & & \\
\hline 10 & 0,002 & 0,016 & 99,998 & & & \\
\hline 11 & 0,000 & 0,002 & 100,000 & & & \\
\hline
\end{tabular}

Fuente: Elaboración propia.

El primer factor está conformado, principalmente, por las variables de cobertura telefónica, cobertura de Internet, establecimientos educacionales, establecimientos de salud y metros cuadrados construidos al año. El segundo componente se concentra en las variables de cobertura de agua potable y cobertura de alcantarillado. A su vez, la variable de caminos de hormigón se agrupa en forma positiva en el tercer factor y la de cobertura de la red eléctrica lo hace en forma negativa. Respecto del cuarto factor, se aprecia que reúne las variables correspondientes a caminos asfaltados y caminos de tierra.

Cuadro 17

Matriz de componentes rotados y comunalidades (dimensión física)

\begin{tabular}{lccccc}
\hline \multirow{2}{*}{ Variables } & \multicolumn{3}{c}{ Componente } & \multicolumn{1}{c}{ C } & \multirow{2}{*}{ Comunalidades } \\
\cline { 2 - 4 } & 1 & 2 & 0,918 & $-0,177$ & 0,878 \\
\hline DF1 & 0,050 & 0,032 & $-0,157$ & 0,864 & 0,887 \\
\hline DF2 & 0,310 & $-0,144$ & 0,525 & 0,491 & 0,877 \\
\hline DF3 & $-0,071$ & $-0,596$ & $-0,448$ & 0,710 & 0,773 \\
\hline DF4 & $-0,247$ & $-0,091$ & 0,030 & $-0,090$ & 0,984 \\
\hline DF5 & 0,970 & 0,183 & 0,042 & $-0,058$ & 0,993 \\
\hline DF6 & 0,976 & 0,188 & 0,157 & $-0,007$ & 0,885 \\
\hline DF7 & 0,033 & 0,927 & $-0,242$ & $-0,155$ & 0,771 \\
\hline DF8 & 0,076 & 0,826 & $-0,704$ & 0,322 & 0,800 \\
\hline DF9 & $-0,362$ & 0,264 & 0,178 & 0,166 & 0,983 \\
\hline DF10 & 0,951 & $-0,137$ & 0,243 & 0,314 & 0,984 \\
\hline DF11 & 0,883 & $-0,218$ & 0,023 & $-0,105$ & 0,974 \\
\hline DF12 & 0,976 & 0,100 & & & \\
\hline
\end{tabular}

Fuente: Elaboración propia. 
Con estos resultados, y dada la alta comunalidad de las variables representada por los componentes seleccionados, se infiere que es posible reducir el número de variables internas de la dimensión. Esto puede lograrse representándolas en constructos que tengan en cuenta el concepto temático que las relaciona, o bien quitando variables redundantes respecto del sentido de la variabilidad que expresan.

\section{iv. Dimensión económica}

La última dimensión sometida al análisis es la dimensión económica, en cuya propuesta inicial se incluyeron las siguientes variables: microempresas (DEc1), pequeñas empresas (DEc2), medianas empresas (DEc3), grandes empresas (DEc4), trabajadores de microempresas (DEc5), trabajadores de pequeñas empresas (DEc6), trabajadores de medianas empresas (DEc7), trabajadores de grandes empresas (DEc8) e inversiones (DEc9).

En esta dimensión se aprecia un alto grado de correlación entre todas las variables, salvo la última (DEc9), que no posee correlaciones significativas con las demás (véase el cuadro 18).

Cuadro 18

Matriz de correlaciones de la dimensión económica

\begin{tabular}{|c|c|c|c|c|c|c|c|c|c|}
\hline Variables & DEc1 & DEc2 & DEc3 & DEc4 & DEc5 & DEc6 & DEc7 & DEC8 & DEc9 \\
\hline DEc1 & 1,000 & & & & & & & & \\
\hline DEc2 & $0,991^{\star * *}$ & 1,000 & & & & & & & \\
\hline DEc3 & $0,978^{\star \star \star}$ & $0,997^{\star \star \star}$ & 1,000 & & & & & & \\
\hline DEc4 & $0,964^{\star \star *}$ & $0,990^{* \star *}$ & $0,998^{\star \star *}$ & 1,000 & & & & & \\
\hline DEc5 & $0,995^{\star \star \star}$ & $0,995^{\star \star \star}$ & $0,985^{\text {*** }}$ & $0,975^{\star \star \star}$ & 1,000 & & & & \\
\hline DEc6 & $0,997^{\star \star \star}$ & $0,997^{\star \star \star}$ & $0,987^{\star \star \star}$ & $0,978^{\star \star \star}$ & $0,998^{\star \star \star}$ & 1,000 & & & \\
\hline $\mathrm{DEc7}$ & $0,986^{\star \star \star}$ & $0,999^{\star \star \star}$ & $0,998^{\star \star *}$ & $0,994^{\star \star *}$ & $0,991^{\star \star \star}$ & $0,994^{\star \star \star}$ & 1,000 & & \\
\hline DEc8 & $0,959^{\star \star \star}$ & $0,987^{\star \star \star}$ & $0,996^{\star \star \star}$ & $0,999^{\star \star \star}$ & $0,971^{\star \star \star}$ & $0,975^{\star \star \star}$ & $0,992^{\star \star \star}$ & 1,000 & \\
\hline DEc9 & 0,277 & 0,361 & 0,399 & 0,420 & 0,318 & 0,307 & 0,373 & 0,431 & 1,000 \\
\hline
\end{tabular}

Fuente: Elaboración propia.

Nota: *Valor $p<0,01 ;{ }^{\star \star}$ valor $p<0,05 ;{ }^{\star \star \star}$ valor $p<0,001$

A su vez, la prueba de esfericidad de Bartlett y la medida de adecuación KMO indican la pertinencia de seguir con el análisis factorial y estudiar la reducción de la dimensionalidad interna de la dimensión (véase el cuadro 19).

Cuadro 19

Prueba de esfericidad de Bartlett y KMO (dimensión económica)

\begin{tabular}{lcr}
\hline Medida de adecuación muestral de Kaiser-Meyer-Olkin & & 0,745 \\
\hline Prueba de esfericidad de Bartlett & Chi-cuadrado aproximado & 495,949 \\
\cline { 2 - 3 } & $\mathrm{gl}$ & 36 \\
\cline { 2 - 3 } & $\mathrm{Sig}$. & 0,000 \\
\hline
\end{tabular}

Fuente: Elaboración propia.

De igual forma, se aprecia que tan solo dos factores permiten explicar un 99,304\% de la varianza total de la dimensión. El primer factor agrupa el 89,940\% de la variabilidad total, mientras que el segundo explica un 9,364\% de la varianza restante de la dimensión en cuestión (véase el cuadro 20). 


\section{Cuadro 20}

Varianza explicada (dimensión económica)

\begin{tabular}{|c|c|c|c|c|c|c|}
\hline \multirow[b]{2}{*}{ Componente } & \multicolumn{3}{|c|}{ Autovalores iniciales } & \multicolumn{3}{|c|}{ Sumas de las saturaciones al cuadrado de la extracción } \\
\hline & Total & $\begin{array}{l}\text { Porcentaje de } \\
\text { la varianza }\end{array}$ & $\begin{array}{l}\text { Porcentaje } \\
\text { acumulado }\end{array}$ & Total & $\begin{array}{c}\text { Porcentaje de } \\
\text { la varianza }\end{array}$ & $\begin{array}{l}\text { Porcentaje } \\
\text { acumulado }\end{array}$ \\
\hline 1 & 8,095 & 89,940 & 89,940 & 8,095 & 89,940 & 89,940 \\
\hline 2 & 0,843 & 9,364 & 99,304 & 0,843 & 9,364 & 99,304 \\
\hline 3 & 0,055 & 0,616 & 99,920 & & & \\
\hline 4 & 0,004 & 0,048 & 99,968 & & & \\
\hline 5 & 0,002 & 0,021 & 99,989 & & & \\
\hline 6 & 0,000 & 0,005 & 99,994 & & & \\
\hline 7 & 0,000 & 0,004 & 99,999 & & & \\
\hline 8 & 0,000 & 0,001 & 100,000 & & & \\
\hline 9 & 0,000 & 0,000 & 100,000 & & & \\
\hline
\end{tabular}

Fuente: Elaboración propia.

Por último, y como era de esperarse dado el análisis de la matriz de correlaciones, las ocho primeras variables asociadas a empresas quedan agrupadas en el primer factor, mientras que el segundo absorbe la variable de inversión (DEc9). De esta forma, se concluye que la dimensión puede explicarse con dos componentes. El primero se refiere a la combinación lineal de las primeras ocho variables, mientras que la variable asociada a la inversión, al modificarse en otra dirección, queda representada por el segundo componente. Cabe destacar el alto valor de las comunalidades. Estas indican que prácticamente la totalidad de la información que contienen las variables queda comprendida en los factores (véase el cuadro 21).

Cuadro 21

Matriz de componentes rotados y comunalidades (dimensión económica)

\begin{tabular}{lccc}
\hline \multirow{2}{*}{ Variables } & \multicolumn{2}{c}{ Componente } & \multirow{2}{*}{ Comunalidades } \\
\cline { 2 - 4 } & 1 & 2 & 0,98 \\
\hline DEc1 & 0,983 & 0,135 & 1,00 \\
\hline DEc2 & 0,978 & 0,209 & 1,00 \\
\hline DEc3 & 0,970 & 0,238 & 0,99 \\
\hline DEc4 & 0,960 & 0,257 & 0,99 \\
\hline DEc5 & 0,982 & 0,160 & 1,00 \\
\hline DEc6 & 0,985 & 0,160 & 1,00 \\
\hline DEc7 & 0,976 & 0,215 & 0,98 \\
\hline DEc8 & 0,957 & 0,263 & 1,00 \\
\hline DEc9 & 0,196 & 0,980 &
\end{tabular}

Fuente: Elaboración propia.

\section{c) Jerarquización de variables}

De manera complementaria al análisis multivariante para la reducción de variables, y como una forma de triangulación metodológica que permitiera integrar los criterios técnicos y teóricos, cualitativos y cuantitativos, se implementó una última etapa de validación. Esa etapa se dedicó a la ponderación de criterios y la jerarquización de variables, basada en el método de jerarquías analíticas de Saaty (1980). 
Este tipo de procedimiento resulta particularmente apropiado cuando es necesario tomar decisiones en torno a la selección de determinados elementos. En este caso, el objetivo de la aplicación era seleccionar un listado final de variables, partiendo de la definición de determinados criterios. Según el método propuesto por Saaty, se establece una matriz de comparación entre pares de criterios y se compara la importancia de cada uno de ellos con los demás. Luego se establece el vector principal, que determina los pesos que a su vez proporcionan una medida cuantitativa de la coherencia de los juicios de valor entre pares de factores (Saaty, 1980).

En términos operativos, y en función de lo sugerido por la metodología, en el procedimiento se consideraron las siguientes etapas:

i) Definición del problema:

¿Cómo seleccionar un conjunto de variables para la construcción de un IDT?

ii) Planteamiento del objetivo:

Jerarquizar un listado de variables para la construcción de un IDT a partir de la definición de criterios y subcriterios de distinta naturaleza.

iii) Definición de criterios y subcriterios:

De acuerdo a la metodología de jerarquías analíticas, se establecieron los criterios y subcriterios que se describen a continuación:

a) Criterio conceptual: referido a los aspectos teóricos y conceptuales del indicador.

- Subcriterio de definición conceptual: si la variable se ajusta a la definición teórica de la dimensión.

- Subcriterio de cobertura temática: si la variable es capaz de tener en cuenta una parte o la totalidad de la definición teórica de la dimensión.

b) Criterio estadístico: referido a los aspectos metodológicos y estadísticos del indicador.

- Subcriterio de análisis factorial: si la variable aporta variabilidad a la dimensión.

- Subcriterio de comparabilidad: si la variable tiene presencia en las distintas comunas y, por ende, es comparable.

- Subcriterio de calidad: si la variable tiene la calidad estadística requerida a nivel comunal.

c) Criterio de gestión: referido al impacto de la política pública en las variaciones del indicador.

- Subcriterio de planificación: si la variable puede verse afectada debido a la intervención de planes o programas a nivel territorial.

- Subcriterio de influencia-dependencia: si la variable ejerce una relación de influencia o dependencia sobre otras variables (o viceversa) del polinomio.

iv) Metodología de evaluación:

La evaluación se llevó a cabo sobre la base de comparaciones pareadas de criterios y subcriterios. La intención es determinar el nivel de importancia de un criterio respecto a otro, de acuerdo a la opinión del evaluador6.

A cada comparación de criterios pareados se le asignó una calificación. En este caso hay más de dos decisores (ocho en total), por lo que el valor final de cada combinación pareada equivaldrá al promedio de las calificaciones otorgadas por cada uno de los decisores, tanto en el primer nivel jerárquico (criterios) como en los subniveles (subcriterios).

6 El panel de expertos está conformado por investigadores (3) del Instituto de Desarrollo Local y Regional de la Universidad de la Frontera y por analistas del Instituto Nacional de Estadísticas en la región de La Araucanía (5). 
El siguiente paso consiste en estimar los pesos relativos de los criterios " $\mathrm{v}$ ", con base en las calificaciones expuestas en la matriz "A". Estos pesos relativos son los vectores propios de la matriz A. El método se basa en el teorema de Perron-Frobenius, que proporciona las prioridades locales mediante la solución del siguiente problema de optimización:

$$
\text { máx } A v=\lambda v \text { s. } a \sum_{j} j v_{j}=1
$$

v) Escala de evaluación:

La evaluación de los criterios se basa en la escala propuesta por Saaty (1980), que cuenta con nueve niveles de valoración. El 1 en dicha escala representa igual importancia de "A respecto a B", y el 9, importancia extrema de "A respecto a B". En el cuadro 22 se presentan con detalle los valores de la escala y sus definiciones.

\section{Cuadro 22}

Escala de valoración del método de jerarquización

\begin{tabular}{lll}
\hline Valor & Definición & Comentarios \\
\hline 1 & Igual importancia & El criterio A es igual de importante que el B \\
\hline 3 & Importancia moderada & La experiencia y el juicio favorecen ligeramente el criterio A sobre el B \\
\hline 5 & Importancia grande & La experiencia y el juicio favorecen decididamente el criterio A sobre el B \\
\hline 9 & Importancia muy grande & El criterio A es mucho más importante que el B, tal como se demuestra en la práctica \\
\hline $2,4,6,8$ & Importancia extrema & Lalores intermedios entre los anteriores, para matizar \\
\cline { 2 - 3 } & Para expresar reciprocidad, se usan los inversos de estos valores \\
\hline
\end{tabular}

Fuente: Elaboración propia.

vi) Resultados de la evaluación:

Una vez llevados a cabo los cálculos y asignados los ponderadores a cada uno de los criterios y subcriterios, se procedió a jerarquizar el listado de variables. De esta forma se descartaron las variables que obtenían bajas puntuaciones en los criterios asignados, lo que significó pasar de un modelo de 49 variables a uno más acotado, compuesto por un total de 19 variables.

\section{Tercer ajuste del modelo}

Al someterse el modelo a las distintas pruebas y metodologías y reducirse la mayor cantidad posible de dimensiones, se logró establecer seis tipos de capital para medir el desarrollo de un territorio. En dichos capitales o dimensiones se incluyen las variables que son más relevantes o explican de mejor forma la dimensión. En el cuadro 23 se describe el tercer ajuste del modelo con las dimensiones y variables necesarias para el cálculo final del índice propuesto. 
Cuadro 23

Matriz para la operacionalización de variables

\begin{tabular}{|c|c|c|c|}
\hline Dimensión & $\begin{array}{l}\text { Identificación de } \\
\text { la variable }\end{array}$ & Definición conceptual & Definición operacional \\
\hline \multirow[t]{4}{*}{ Capital cultural } & $\begin{array}{l}\text { 1. Patrimonio cultural } \\
\text { material }\end{array}$ & $\begin{array}{l}\text { Lugares, sitios, edificaciones, obras de ingeniería, centros } \\
\text { industriales, conjuntos arquitectónicos, zonas típicas y } \\
\text { monumentos de interés o valor relevante desde el punto } \\
\text { de vista arquitectónico, arqueológico, histórico, artístico } \\
\text { o científico, reconocidos y registrados como tales. }\end{array}$ & $\begin{array}{l}\text { Número de hitos } \\
\text { patrimoniales declarados } \\
\text { por decreto, por comuna. }\end{array}$ \\
\hline & $\begin{array}{l}\text { 2. Patrimonio cultural } \\
\text { inmaterial }\end{array}$ & $\begin{array}{l}\text { Tradiciones o expresiones vivas, heredadas de los } \\
\text { antepasados y transmitidas a los descendientes, por } \\
\text { ejemplo: tradiciones orales, artes del espectáculo, } \\
\text { usos sociales, rituales, actos festivos, conocimientos y } \\
\text { prácticas relativos a la naturaleza y el universo, así como } \\
\text { saberes y técnicas vinculados a la artesanía tradicional. }\end{array}$ & $\begin{array}{l}\text { Número de hitos patrimoniales } \\
\text { inmateriales registrados en } \\
\text { el SIGPA, por comuna. }\end{array}$ \\
\hline & $\begin{array}{l}\text { 3. Comunidades } \\
\text { y asociaciones } \\
\text { indígenas }\end{array}$ & $\begin{array}{l}\text { Se entiende por comunidad indígena toda agrupación } \\
\text { de personas pertenecientes a una misma etnia, que se } \\
\text { encuentren en una o más de las siguientes situaciones: } \\
\text { i) provengan de un mismo tronco familiar; ii) reconozcan } \\
\text { una jefatura tradicional; iii) posean o hayan poseído } \\
\text { tierras indígenas en común, y iv) provengan de un } \\
\text { mismo poblado antiguo. Se entiende por asociación } \\
\text { indígena la agrupación voluntaria y funcional integrada, } \\
\text { al menos, por } 25 \text { indígenas que se congregan en } \\
\text { función de algún interés y objetivo común. }\end{array}$ & $\begin{array}{l}\text { Número de comunidades } \\
\text { y asociaciones indígenas, } \\
\text { por comuna. }\end{array}$ \\
\hline & $\begin{array}{l}\text { 4. Marcas territoriales } \\
\text { y denominaciones } \\
\text { de origen }\end{array}$ & $\begin{array}{l}\text { Las indicaciones geográficas y las denominaciones } \\
\text { de origen protegen productos originarios del país } \\
\text { o de una región o localidad, siempre que tengan } \\
\text { una calidad, reputación u otra característica } \\
\text { imputable a su origen geográfico. }\end{array}$ & $\begin{array}{l}\text { Número de indicaciones } \\
\text { geográficas y denominaciones } \\
\text { de origen, por comuna. }\end{array}$ \\
\hline \multirow[t]{2}{*}{ Capital social } & $\begin{array}{l}\text { 5. Agrupaciones con } \\
\text { fines sociales }\end{array}$ & $\begin{array}{l}\text { Agrupación de interés público sin fines de lucro cuya } \\
\text { finalidad es promover el interés general, ya sea en } \\
\text { materia de derechos ciudadanos, asistencia social, } \\
\text { educación, salud, medio ambiente, o cualquier otro } \\
\text { asunto de bien común. (Los centros de madres, de } \\
\text { padres y de apoderados, los centros u organizaciones } \\
\text { de adultos mayores, los clubes deportivos, las juntas } \\
\text { de vecinos y otras agrupaciones funcionales). }\end{array}$ & $\begin{array}{l}\text { Número de agrupaciones con } \\
\text { fines sociales, con personalidad } \\
\text { jurídica vigente, por comuna. }\end{array}$ \\
\hline & 6. Participación electoral & $\begin{array}{l}\text { Votantes inscritos (en porcentajes), que participan } \\
\text { voluntariamente en las elecciones municipales. }\end{array}$ & $\begin{array}{l}\text { Porcentaje de votantes respecto } \\
\text { del total de inscritos, por comuna. }\end{array}$ \\
\hline \multirow[t]{3}{*}{ Capital humano } & 7. Años de escolaridad & $\begin{array}{l}\text { Promedio de años cursados en el sistema educacional. } \\
\text { Se aplica a las personas de } 15 \text { años y más. }\end{array}$ & $\begin{array}{l}\text { Años de escolaridad } \\
\text { media, por comuna. }\end{array}$ \\
\hline & $\begin{array}{l}\text { 8. Pruebas de selección } \\
\text { universitaria (PSU) }\end{array}$ & $\begin{array}{l}\text { Puntaje medio obtenido en las pruebas de } \\
\text { selección universitaria, entre las pruebas } \\
\text { de Lenguaje y Matemáticas. }\end{array}$ & $\begin{array}{l}\text { Promedio obtenido en las } \\
\text { PSU, por comuna. }\end{array}$ \\
\hline & $\begin{array}{l}\text { 9. Profesionalización del } \\
\text { personal municipal }\end{array}$ & $\begin{array}{l}\text { Capital humano contratado por el municipio que } \\
\text { cuenta con grado o calificación profesional. }\end{array}$ & $\begin{array}{l}\text { Porcentaje de trabajadores } \\
\text { profesionales con que cuenta } \\
\text { el municipio, por comuna. }\end{array}$ \\
\hline \multirow[t]{3}{*}{ Capital institucional } & $\begin{array}{l}\text { 10. Dependencia del } \\
\text { Fondo Común } \\
\text { Municipal (FCM) }\end{array}$ & $\begin{array}{l}\text { Dependencia del FCM (como principal fuente de } \\
\text { financiamiento) respecto a los ingresos generados } \\
\text { por el propio municipio. El FCM es un mecanismo de } \\
\text { redistribución solidaria de los recursos financieros entre } \\
\text { las municipalidades del país, tendiente a garantizar el } \\
\text { cumplimiento de los fines y el adecuado funcionamiento } \\
\text { de los municipios. El FCM fue ideado para beneficiar } \\
\text { a los distritos de menores recursos, en especial, los } \\
\text { que tienen escasas posibilidades de acceder a otros } \\
\text { ingresos propios permanentes, por lo que constituye } \\
\text { la principal fuente de ingresos municipales. }\end{array}$ & $\begin{array}{l}\text { Porcentaje del ingreso total } \\
\text { municipal proveniente del } \\
\text { FCM, por municipio. }\end{array}$ \\
\hline & 11. Gastos municipales & $\begin{array}{l}\text { Partida del presupuesto municipal que incluye } \\
\text { gastos de operación, de transferencia y de } \\
\text { inversión, aplicación de ingresos de terceros y otros } \\
\text { gastos, divididos entre la población comunal. }\end{array}$ & $\begin{array}{l}\text { Gastos municipales devengados } \\
\text { per cápita en pesos } \\
\text { chilenos, por municipio. }\end{array}$ \\
\hline & $\begin{array}{l}\text { 12. Ordenanzas } \\
\text { municipales }\end{array}$ & $\begin{array}{l}\text { Las ordenanzas son normas generales y obligatorias } \\
\text { aplicables a la comunidad. En ellas podrán } \\
\text { establecerse multas cuyo monto no excederá de } \\
\text { cinco unidades tributarias mensuales, aplicadas por } \\
\text { los juzgados de policía local correspondientes. }\end{array}$ & $\begin{array}{l}\text { Número de ordenanzas } \\
\text { municipales, por comuna. }\end{array}$ \\
\hline
\end{tabular}


Cuadro 23 (conclusión)

\begin{tabular}{|c|c|c|c|}
\hline Dimensión & $\begin{array}{l}\text { Identificación de } \\
\text { la variable }\end{array}$ & Definición conceptual & Definición operacional \\
\hline \multirow[t]{4}{*}{ Capital físico } & 13. Red vial & $\begin{array}{l}\text { Los caminos de hormigón son lozas de concreto, } \\
\text { separadas por juntas y colocadas sobre una } \\
\text { base granular debidamente compactada. Los } \\
\text { caminos de asfalto, por su parte, son capas de } \\
\text { rodadura de concreto asfáltico sin juntas, que } \\
\text { no deben tener menos de } 10 \mathrm{~cm} \text { de espesor. }\end{array}$ & $\begin{array}{l}\text { Kilómetros de caminos de } \\
\text { hormigón y asfalto, expresados } \\
\text { como porcentaje del total } \\
\text { de caminos, por comuna. }\end{array}$ \\
\hline & $\begin{array}{l}\text { 14. Penetración } \\
\text { de Internet }\end{array}$ & $\begin{array}{l}\text { Parte de la población conectada a una } \\
\text { red fija de Internet domiciliaria. }\end{array}$ & $\begin{array}{l}\text { Porcentaje de cobertura } \\
\text { Internet, por comuna. }\end{array}$ \\
\hline & $\begin{array}{l}\text { 15. Metros cuadrados } \\
\text { construidos }\end{array}$ & $\begin{array}{l}\text { Metros cuadrados construidos con recepción definitiva } \\
\text { durante el año. Están exentas de regularización } \\
\text { las construcciones anteriores a la Ley General de } \\
\text { Construcciones y Urbanización, de mayo de } 1931 \text {. }\end{array}$ & $\begin{array}{l}\text { Porcentaje estimado } \\
\text { de construcciones con } \\
\text { recepción definitiva sobre } \\
\text { el total de construcciones } \\
\text { existentes en la comuna. }\end{array}$ \\
\hline & $\begin{array}{l}\text { 16. Cobertura de agua } \\
\text { potable rural }\end{array}$ & $\begin{array}{l}\text { Parte de la población rural que cuenta con } \\
\text { suministro de agua potable en la propia vivienda o } \\
\text { en el terreno, o de una llave pública o hidrante. }\end{array}$ & $\begin{array}{l}\text { Porcentaje de cobertura agua } \\
\text { potable rural, por comuna. }\end{array}$ \\
\hline \multirow[t]{3}{*}{ Capital económico } & $\begin{array}{l}\text { 17. Remuneración de } \\
\text { los trabajadores }\end{array}$ & $\begin{array}{l}\text { Remuneraciones medias de los trabajadores } \\
\text { dependientes informados por el Servicio de } \\
\text { Impuestos Internos (SII), estimadas a partir de las } \\
\text { remuneraciones y el número de trabajadores. }\end{array}$ & $\begin{array}{l}\text { Salario medio mensual } \\
\text { en pesos chilenos de los } \\
\text { trabajadores dependientes } \\
\text { informados, por comuna. }\end{array}$ \\
\hline & $\begin{array}{l}\text { 18. Inversión del } \\
\text { Fondo Nacional } \\
\text { de Desarrollo } \\
\text { Regional (FNDR) }\end{array}$ & Ejecución de programas de inversión regional. & $\begin{array}{l}\text { Gasto devengado anual per cápita } \\
\text { en pesos chilenos, por comuna. }\end{array}$ \\
\hline & $\begin{array}{l}\text { 19. Colocaciones } \\
\text { bancarias }\end{array}$ & $\begin{array}{l}\text { La colocación o crédito es un préstamo en efectivo } \\
\text { que un banco otorga a su cliente, con el compromiso } \\
\text { de que, en el futuro, el cliente lo devolverá en forma } \\
\text { gradual, mediante el pago de cuotas, o en un solo pago } \\
\text { y con un interés adicional que compense al acreedor } \\
\text { por el período en que no dispuso de ese dinero. }\end{array}$ & $\begin{array}{l}\text { Número de colocaciones } \\
\text { per cápita, por comuna. }\end{array}$ \\
\hline
\end{tabular}

Fuente: Elaboración propia.

\section{a) Ponderación del índice de desarrollo territorial}

Se ha utilizado el criterio de equiponderación de dimensiones para asignar los pesos relativos de las dimensiones y de las respectivas variables internas de las dimensiones. Se han tomado como referencia los criterios utilizados por el PNUD/MIDEPLAN (2005) en la asignación de ponderadores del índice de desarrollo humano (IDH) comunal y en la nueva metodología para la medición de la pobreza multidimensional en Chile, propuesta por el Observatorio Social del MIDESO. La equiponderación significa que las dimensiones tienen el mismo peso específico dentro del índice y que a las variables se les aplica la misma ponderación dentro de la dimensión.

Según Alkire y Foster (2011) la selección de ponderadores puede considerarse como un juicio de valor, abierto al escrutinio público. No existe una fórmula definitiva para la asignación de ponderadores. Sin embargo, en términos generales, hay al menos dos alternativas igualmente válidas: i) dar igual peso a las variables, lo que podría significar que las dimensiones tengan pesos diferentes si el número de variables por dimensión es distinto, o ii) dar igual peso a las dimensiones, lo que podría implicar que las variables tengan ponderaciones diferentes, si el número de variables por dimensiones es distinto. En el caso del índice que se propone, se aplica la segunda opción.

De conformidad con la premisa en que se basa la ponderación equivalente o equiponderada, no existen argumentos de consenso que justifiquen o avalen una asignación de ponderadores distintos a las distintas dimensiones, o variables, según corresponda (Ministerio de Desarrollo Social, 2013). En lo que se refiere a la ponderación de escalas actitudinales, Canales (2006) señala que, si no hay ninguna razón teórica o empírica contundente para asignar mayor peso a una pregunta o a otra, lo mejor es que todas pesen igual. Así, por ejemplo, el papel que juega el capital institucional en este caso sería tan importante como el del capital físico o social en la articulación de los procesos de desarrollo territorial. 
El IDT que se propone en este trabajo está compuesto por un total de seis dimensiones (capitales), por lo que cada dimensión tendrá una ponderación del 16,6\%. Como ya se ha indicado, la ponderación de cada variable dependerá del número de variables por dimensión. En el cuadro 24 se describe con detalle la ponderación del índice.

Cuadro 24

Estructura de ponderadores

\begin{tabular}{|c|c|c|c|}
\hline Capital & $\begin{array}{l}\text { Peso de la dimensión } \\
\text { (en porcentajes) }\end{array}$ & Identificación de la variable & $\begin{array}{l}\text { Peso de la variable } \\
\text { (en porcentajes) }\end{array}$ \\
\hline \multirow[t]{4}{*}{ 1. Cultural } & 16,67 & 1. Patrimonio cultural material & 4,17 \\
\hline & & 2. Patrimonio cultural inmaterial & \\
\hline & & 3. Comunidades y asociaciones indígenas & \\
\hline & & 4. Marcas territoriales y denominaciones de origen & \\
\hline \multirow[t]{2}{*}{ 2. Social } & 16,67 & 5. Agrupaciones con fines sociales & 8,33 \\
\hline & & 6. Participación electoral & \\
\hline \multirow[t]{3}{*}{ 3. Humano } & 16,67 & 7. Años de escolaridad & 5,56 \\
\hline & & 8. Pruebas de selección universitaria (PSU) & \\
\hline & & 9. Profesionalización del personal municipal & \\
\hline \multirow[t]{3}{*}{ 4. Institucional } & 16,67 & 10. Dependencia del FCM & 5,56 \\
\hline & & 11. Gastos municipales & \\
\hline & & 12. Ordenanzas municipales & \\
\hline \multirow[t]{4}{*}{ 5. Físico } & 16,67 & 13. Red vial & 4,17 \\
\hline & & 14. Penetración de Internet & \\
\hline & & 15. Metros cuadrados construidos & \\
\hline & & 16. Cobertura de agua potable rural & \\
\hline \multirow[t]{3}{*}{ 6. Económico } & 16,67 & 17. Remuneración de los trabajadores & 5,56 \\
\hline & & 18. Inversión del FNDR & \\
\hline & & 19. Colocaciones bancarias & \\
\hline
\end{tabular}

Fuente: Elaboración propia.

\section{b) Descripción del índice de desarrollo territorial}

El índice de desarrollo territorial (IDT) es un indicador que permite reunir un conjunto de variables disímiles, tanto en escala como en unidades de medida, agrupadas en seis tipos de capital territorial. Con esas variables se pretende dar cuenta de los principales fenómenos asociados a la capacidad de desarrollo, no solo a partir del componente económico, sino de todos los aspectos que interaccionan en el territorio, por ejemplo, el capital cultural, social, humano, entre otros.

Como se ha mencionado ampliamente, los factores de desarrollo incorporados en la medición son: capital cultural, capital social, capital humano, capital institucional, capital físico y capital económico. En su conjunto, agrupan un total de 19 variables. Así, de acuerdo al apartado anterior, cada uno de los factores o capitales involucrados está equiponderado, y su carga se distribuye igualitariamente entre las variables que sirven de soporte a cada una de las dimensiones.

Es decir, el peso específico de cada dimensión quedará determinado por la constante $w=\frac{100}{n}$, en la que $n$ equivale a la cantidad de dimensiones. Entretanto, en cada dimensión la ponderación quedará explicitada de la siguiente forma: $\alpha_{i}=\frac{100}{n * m_{i}}$, donde $m_{i}$ hace referencia al número de variables dentro de la i-ésima dimensión $(i=1,2, \ldots, n)$.

Ahora bien, dada la diversidad de escalas de las variables en cuestión, y de acuerdo a las múltiples alternativas en la estandarización de datos, se optó por aplicar el método de mínimos y máximos, procedimiento utilizado por el PNUD en la estandarización del IDH. Según lo expresado 
por la oficina del PNUD en Chile, al establecer pisos y metas fijos, la lógica de los valores mínimos y máximos normativos permite que cada país, región y comuna se comparen consigo mismos. Esta metodología permite, por ejemplo, que el valor absoluto del IDH de una unidad analizada no dependa del desempeño del resto y que sea una herramienta útil más allá de toda comparación relativa (PNUD/MIDEPLAN, 2005). Así pues, tomando en consideración lo anteriormente expuesto, se optó por llevarlas a una graduación única y común, que variara entre 0 y 1.

Esto se logra mediante el cálculo de la razón entre la distancia efectiva y el rango de variabilidad correspondiente a cada variable. Es decir, para la $j$-ésima variable se adopta la siguiente transformación:

$$
Z_{j}=\frac{X_{j}-X_{j}^{\min }}{X_{j}^{\text {máx }}-X_{j}^{m i n}}
$$

Donde $X_{j}^{\min }=\min \left(X_{j}^{k}, k=1, \ldots, 342\right)$ y $X_{j}^{\text {máx }}=\operatorname{máx}\left(X_{j}^{k}, k=1, \ldots, 342\right)$. Por último, el cálculo del índice en cada factor adopta la siguiente estructura:

$$
I_{i}=\alpha_{i} \sum_{j=1}^{m_{i}} Z_{j}
$$

De esta forma, el índice general se puede calcular tomando la suma ponderada de los índices por factores (de manera equivalente, se puede tomar la suma ponderada a través de las variables). El IDT quedará determinado por la siguiente relación funcional:

$$
I=w \sum_{i=1}^{n} I_{i}
$$

Así, el rango de variabilidad del indicador oscila entre 0 y 1 . Los valores más cercanos a la unidad denotan mayor desarrollo del territorio y, a la inversa, los valores cercanos a 0 dan inferencia de un menor nivel de desarrollo. Los componentes de los factores ponen de relieve las posibilidades y potencialidades que se podrían explotar en ellos.

\section{c) Cálculo del índice de desarrollo territorial}

A continuación se presentan los resultados del cálculo del indicador en las comunas indicadas, es decir, Angol y Carahue (véase el cuadro 21).

Según los resultados del IDT, la comuna de Angol se ubica en un nivel de desarrollo superior al de la comuna de Carahue. En términos numéricos, Angol presenta un IDT de 0,280, mientras que Carahue muestra un valor de 0,232, con una brecha de desarrollo de 0,048 puntos.

Esta diferencia se puede explicar mediante el análisis de los resultados referentes a los capitales territoriales. Como se aprecia en el gráfico 1, a excepción del capital económico, Angol posee mayor desarrollo en todos los factores de medición. Las mayores diferencias se observan en el capital humano (0,096 puntos), el capital cultural (0,089 puntos) y el capital físico (0,066 puntos).

En la dimensión del capital humano, Angol presenta mejores características que Carahue, debido a un mejor posicionamiento en las variables referentes a los años de escolaridad y las PSU. No obstante, la comuna de Carahue presenta un mayor porcentaje de profesionalización del personal municipal. 


\section{Gráfico 1}

Índice de desarrollo territorial por dimensiones, comunas de Angol y Carahue

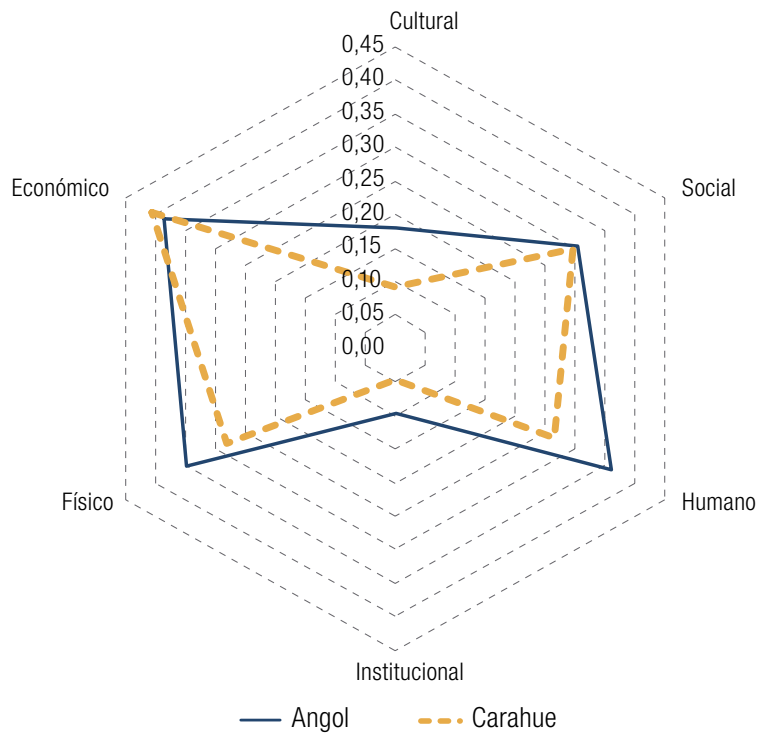

Fuente: Elaboración propia, sobre la base de estadísticas oficiales.

Respecto del capital cultural, el resultado comparativo se define principalmente por la mayor cuantía de marcas territoriales en Angol, así como por el mayor número de hitos registrados como patrimonio cultural inmaterial. En la comuna de Carahue, estas diferencias se compensan en parte gracias a la predominación de comunidades indígenas, así como al patrimonio cultural material.

En cuanto al capital físico, nuevamente Angol presenta mejor situación que Carahue, en atención a que la primera tiene mayor penetración de Internet y mayor superficie de infraestructura construida. Sin embargo, la comuna de Carahue cuenta con un mayor porcentaje de caminos de hormigón y asfalto en relación con el total de la red de caminos comunales.

En lo que respecta al capital institucional, se observa que la comuna de Angol está en mejores condiciones que la de Carahue. Esto se debe a la menor dependencia del fondo común municipal, el mayor gasto municipal per cápita y la mayor cantidad de ordenanzas municipales.

En la dimensión referida al capital social no se aprecian diferencias significativas entre Carahue y Angol debido a la mayor participación en elecciones municipales en Carahue, aunque esta comuna tenga un menor número de agrupaciones sociales (véase el cuadro 25).

Por último, en cuanto al capital económico, Carahue presenta mejores cifras que Angol, al ostentar una mayor remuneración media de los trabajadores y un mayor volumen de inversión delegada del Fondo Nacional de Desarrollo Regional (FNDR) debido a los importantes proyectos de inversión desarrollados durante los últimos tiempos. No obstante, a nivel de colocaciones de la banca en las comunas, Angol presenta mejores cifras que Carahue, lo que podría deberse a que la primera tiene una mayor cantidad de población bancarizable que la segunda. 
Cuadro 25

Cálculo del índice de desarrollo territorial

\begin{tabular}{|c|c|c|c|c|c|c|c|c|c|}
\hline Capital & Identificación de la variable & Angol & Carahue & Min & Máx & ZAngol & ZCarahue & Angol & Carahue \\
\hline \multirow[t]{4}{*}{ Cultural } & $\begin{array}{l}\text { 1. Patrimonio cultural } \\
\text { material }\end{array}$ & 6,0 & 32,0 & 0,0 & 190,0 & 0,03 & 0,17 & 0,18 & 0,09 \\
\hline & $\begin{array}{l}\text { 2. Patrimonio cultural } \\
\text { inmaterial }\end{array}$ & 8,0 & 5,0 & 0,0 & 61,0 & 0,13 & 0,08 & & \\
\hline & $\begin{array}{l}\text { 3. Comunidades y } \\
\text { asociaciones indígenas }\end{array}$ & 0,7 & 1,4 & 0,0 & 11,8 & 0,06 & 0,11 & & \\
\hline & $\begin{array}{l}\text { 4. Marcas territoriales y } \\
\text { denominaciones de origen }\end{array}$ & 1,0 & 0,0 & 0,0 & 2,0 & 0,50 & 0,00 & & \\
\hline \multirow[t]{2}{*}{ Social } & $\begin{array}{l}\text { 5. Agrupaciones con } \\
\text { fines sociales }\end{array}$ & 692,0 & 148,0 & 0,0 & 4305,0 & 0,16 & 0,03 & 0,30 & 0,30 \\
\hline & 6. Participación electoral & 50,6 & 56,7 & 26,8 & 80,0 & 0,45 & 0,56 & & \\
\hline \multirow[t]{3}{*}{ Humano } & 7. Años de escolaridad & 10,0 & 8,0 & 7,0 & 16,0 & 0,33 & 0,11 & 0,36 & 0,27 \\
\hline & 8. PSU & 469,8 & 435,6 & 362,4 & 613,5 & 0,43 & 0,29 & & \\
\hline & $\begin{array}{l}\text { 9. Profesionalización del } \\
\text { personal municipal }\end{array}$ & 0,2 & 0,3 & 0,0 & 0,7 & 0,32 & 0,39 & & \\
\hline \multirow[t]{3}{*}{ Institucional } & 10. Dependencia del FCM & 0,7 & 0,9 & 1,0 & 0,0 & 0,26 & 0,11 & 0,10 & 0,05 \\
\hline & 11. Gastos municipales & 191,0 & 180,4 & 65,8 & 10531,1 & 0,01 & 0,01 & & \\
\hline & 12. Ordenanzas municipales & 16,0 & 13,0 & 2,0 & 609,0 & 0,02 & 0,02 & & \\
\hline \multirow[t]{4}{*}{ Físico } & 13. Red vial & 11,2 & 14,4 & 0,0 & 100,0 & 0,11 & 0,14 & 0,35 & 0,28 \\
\hline & 14. Penetración de Internet & 22,9 & 7,1 & 0,0 & 100,6 & 0,23 & 0,07 & & \\
\hline & $\begin{array}{l}\text { 15. Metros cuadrados } \\
\text { construidos }\end{array}$ & 2,1 & 0,9 & 0,0 & 24,1 & 0,09 & 0,04 & & \\
\hline & $\begin{array}{l}\text { 16. Cobertura de agua } \\
\text { potable rural }\end{array}$ & 1,0 & 0,9 & 0,0 & 1,0 & 0,97 & 0,88 & & \\
\hline \multirow[t]{3}{*}{ Económico } & $\begin{array}{l}\text { 17. Remuneración de } \\
\text { los trabajadores }\end{array}$ & 185833 & 333223 & 46164 & 1703167 & 0,39 & 0,55 & 0,39 & 0,41 \\
\hline & 18. Inversión del FNDR & 32,1 & 85,9 & 0,0 & 4950,3 & 0,01 & 0,02 & & \\
\hline & 19. Colocaciones bancarias & 1,8 & 0,2 & 0,0 & 151,2 & 0,77 & 0,66 & & \\
\hline Total & & & & & & & & 0,2802 & 0,2319 \\
\hline
\end{tabular}

Fuente: Elaboración propia, sobre la base de estadísticas oficiales.

\section{Conclusiones}

El presente artículo ha sido fruto de un extenso trabajo y largas jornadas de discusión de un equipo multidisciplinario. Durante tres años, los investigadores se dieron a la tarea de diseñar un índice de desarrollo que tuviera en cuenta cómo el aprovechamiento de determinados tipos de capital puede dar lugar a procesos de desarrollo territorial en el ámbito local. Al desafío anterior se debe sumar la complejidad asociada a la escasez de información disponible a nivel subregional. Ello entrañó un arduo trabajo de revisión, sistematización y selección de fuentes de datos secundarias que, además de dar cobertura temática al modelo, respondieran a criterios de representatividad, comparabilidad, periodicidad, entre otros. Esto se hizo con el propósito de dejar a disposición de los gestores locales un sistema de medición del desarrollo que fuera lo suficientemente práctico para reproducirlo a lo largo del tiempo.

Además de todo lo anterior, existen otros elementos que refuerzan el trabajo realizado. Al respecto, cabe destacar la estrecha relación que se establece entre los niveles teórico, analítico y práctico. Se trata de una propuesta que parte de la articulación de un modelo teórico (de tipos de capital territorial). Este modelo recoge elementos de las distintas teorías del desarrollo que definen las dimensiones del índice. Luego se procede a una serie de análisis estadísticos multivariantes con que se busca lograr ciertos ajustes del modelo a partir de la reducción de la dimensionalidad de los datos (y validar técnicamente la propuesta), hasta llegar al cálculo simplificado de un IDT respecto de las comunas de Angol y Carahue, sobre la base de registros administrativos. 
Además, conviene recalcar la importancia que se ha dado en este trabajo al registro de la trastienda de la investigación. Es decir, más allá de mostrar únicamente los resultados del cálculo final del indicador, se ha intentado documentar las distintas etapas y las decisiones metodológicas para introducir ajustes en el indicador, hasta llegar a una versión (más o menos) definitiva. Lo anterior es relevante en la medida en que esta práctica permite a otros investigadores conocer más detalles internos del proceso, con inclusión de los procedimientos implicados, las determinaciones y los aciertos y desaciertos. En definitiva, este tipo de enfoque metodológico fomenta el debate abierto en torno a cómo se van adquiriendo los conocimientos necesarios en un determinado campo científico.

El sentido final de la construcción de un indicador de esta naturaleza radica en la posibilidad de facilitar información detallada para la toma de decisiones. En primer lugar, se busca diagnosticar los factores basales o estructurales que posibilitan el desarrollo. En segundo lugar, se intenta proporcionar información respecto de las variables en que es posible influir mediante las decisiones de política pública o privada, a fin de mejorar las condiciones de desarrollo (desde una perspectiva multidimensional) de dicho espacio territorial.

Otro aporte sustantivo se refiere a los enfoques teóricos asociados a la identificación de factores estructurales que definen la capacidad de un espacio territorial de tomar en cuenta la complejidad del desarrollo. Se concluye que el enfoque de los tipos de capital territorial ofrece un sustento basal que permite profundizar en los análisis realizados por Alburquerque, Vásquez Barquero, Boisier, Requejo e instituciones como el PNUD. Esto representa una contribución, por ejemplo, a las deliberaciones asociadas a la política de gestión territorial de las zonas de rezago en Chile.

\section{Bibliografía}

Alburquerque, F. (2013), "Economía del desarrollo y desarrollo territorial” [en línea] http://www.conectadel. org/wp-content/uploads/downloads/2015/03/E\%C2\%AADesarrollo-y-Desarrollo-Territorial-3.01.pdf.

Alkire, S. y J. Foster (2011), "Understandings and misunderstandings of multidimensional poverty measurement", Journal of Economic Inequality, vol. 9, No 2, Springer.

Boisier, S. (2004), "Una (re)visión heterodoxa del desarrollo (territorial): un imperativo categórico", Estudios Sociales, vol. 12, № 23, enero-junio [en línea] https://dialnet.unirioja.es/descarga/articulo/2108278.pdf.

Canales, M. (2006), Metodologías de investigación social. Introducción a los oficios, Santiago, LOM Ediciones.

Cea D’Ancona, M. A. (2002), Análisis multivariable. Teoría y práctica en la investigación social, Madrid, Síntesis.

Cerda, T. y X. Vera (2008), "Indicadores sociales y marcos conceptuales para la medición social", Documento de Trabajo, Santiago, Instituto Nacional de Estadísticas (INE) [en línea] http://historico.ine.cl/canales/sala_prensa/ revistaseconomicas/documentostrabajo/30_06_09/documento_de_trabajo_indicadores_sociales.pdf.

Chile Emprende (2005), Guía metodológica del desarrollo territorial, Santiago [en línea] http://www.dhl.hegoa. ehu.es/ficheros/0000/0243/Chile_Estrategia_de_desarrollo_econ\%C3\%B3mico_territorial_2009.pdf.

Comisión Asesora Presidencial en Descentralización y Desarrollo Regional (2014), "Propuesta de política de Estado y agenda para la descentralización y el desarrollo territorial de Chile. Hacia un país desarrollado y justo", Santiago [en línea] http://www.senado.cl/prontus_senado/site/artic/20141007/asocfile/20141007112343/ descentralizacion_informe.pdf.

Cuadras, C. M. (2007), Nuevos métodos de análisis mutivariante, Barcelona, CMC Editions [en línea] http:// www.est.uc3m.es/esp/nueva_docencia/getafe/estadistica/analisis_multivariante/doc_generica/archivos/ metodos.pdf.

ECOSOC (Consejo Económico y Social) (2011), "Informe de Francia sobre la medición de los resultados económicos y el progreso social" (E/CN.3/2011/35), Naciones Unidas [en línea] https://unstats.un.org/ unsd/statcom/doc11/2011-35-France-S.pdf.

Figueras, M. (2001), "Análisis de conglomerados o cluster" [en línea] http://ciberconta.unizar.es/LECCION/ cluster/100.HTM. 
Filgueira, F. (2006), "La antipática pero necesaria defensa política de la tecnocracia en América Latina", Evaluación para el desarrollo social: aportes para un debate abierto en América Latina, M. Vera (ed.), Ciudad de Guatemala, Magna Terra Editores S.A.

García, E., J. Gil y G. Rodríguez (2001), "Análisis factorial”, Cuadernos de Estadística, № 7, Madrid, Editorial Hespérides/Editorial La Muralla.

González, H., O. M. Sánchez e Y. Araúz (2011), "Marco conceptual, metodológico y operativo de la planificación regional: guía metodológica para el diseño de la estrategia de abordaje regional en planificación del desarrollo", Ministerio de Planificación Nacional y Política Económica (MIDEPLAN)/Programa de las Naciones Unidas para el Desarrollo (PNUD) [en línea] https://documentos.mideplan.go.cr/alfresco/d/d/ workspace/SpacesStore/8c656860-8f17-47df-a6e1-67cfccf89dff/CPR-001_1.pdf?guest=true.

Hair, J. F., R. E. Anderson y R. L. Tatham (1999), Análisis multivariante, Madrid, Prentice Hall.

Ministerio de Desarrollo Social (2013), "Procedimiento de cálculo de la tasa de pobreza a nivel comunal mediante la aplicación de metodología de estimación para áreas pequeñas (SAE)", Serie Documentos Metodológicos, № 1 [en línea] http://observatorio.ministeriodesarrollosocial.gob.cl/indicadores/docs/ Procedimiento_de_calculo_de_la_Tasa_de_Pobreza_a_nivel_Comunal_11feb13_5118dab432f1c.pdf.

OCDE (Organización de Cooperación y Desarrollo Económicos) (2008), Handbook on Constructing Composite Indicators: Methodology and User Guide, París [en línea] http://www.oecd.org/sdd/42495745.pdf.

PNUD/MIDEPLAN (Programa de las Naciones Unidas para el Desarrollo/Ministerio de Planificación y Cooperación) (2005), Las trayectorias del desarrollo humano en las comunas de Chile (1994-2003), Santiago [en línea] http://desarrollohumano.cl/idh/download/IDHC\%20con\%20portada.pdf.

Requejo, J. (2007), "Clases de capital territorial. Clases de capital territorial y desarrollo sostenible" [en línea] http://www.atclave.es/publicaciones/descargas/pub_desarrollo/17_clases_de_capital_territorial.pdf.

Saaty, T. (1980), The Analytic Hierarchy Process, Nueva York, McGraw Hill.

Schuschny, A. y H. Soto (2009), "Guía metodológica: diseño de indicadores compuestos de desarrollo sostenible", Documento de Proyecto (LC/W.255), Santiago, Comisión Económica para América Latina y el Caribe (CEPAL) [en línea] http://repositorio.cepal.org/bitstream/handle/11362/3661/1/S2009230_es.pdf.

Toloza, I. (2007), "Desarrollo territorial: un modelo descentralizado de gestión en la región de la Araucanía, Chile", tesis, Universidad Austral de Chile.

Vázquez Barquero, A. (2007), "Desarrollo endógeno. Teorías y políticas de desarrollo territorial”, Investigaciones Regionales, № 11, Madrid, Asociación Española de Ciencia Regional [en línea] http://www.redalyc.org/ pdf/289/28901109.pdf. 


\section{Anexo A1}

\section{Cuadro A1.1}

Primera identificación de variables por dimensión

\begin{tabular}{|c|c|}
\hline \multicolumn{2}{|l|}{ Variables identificadas } \\
\hline \multirow[t]{10}{*}{ Dimensión cultural } & 1. Número de lof \\
\hline & 2. Número de comunidades indígenas \\
\hline & 3. Número de agrupaciones indígenas \\
\hline & 4. Kilómetros de áreas de desarrollo indígena \\
\hline & 5. Número de machis \\
\hline & 6. Número de loncos \\
\hline & 7. Número de hitos patrimoniales \\
\hline & 8. Número de denominaciones de origen \\
\hline & 9. Número de marcas territoriales \\
\hline & 10. Número de eventos culturales \\
\hline \multirow[t]{17}{*}{ Dimensión social } & 11. Número de ONG \\
\hline & 12. Número de juntas de vecinos \\
\hline & 13. Número de uniones comunales \\
\hline & 14. Número de mesas territoriales \\
\hline & 15. Número de clubes de adultos mayores \\
\hline & 16. Número de agrupaciones juveniles \\
\hline & 17. Número de agrupaciones deportivas \\
\hline & 18. Número de agrupaciones culturales \\
\hline & 19. Número de agrupaciones de salud \\
\hline & 20. Número de agrupaciones de mujeres \\
\hline & 21. Número de iglesias \\
\hline & 22. Número de agrupaciones de beneficencia \\
\hline & 23. Número de cooperativas \\
\hline & 24. Número de asociaciones de empresarios \\
\hline & 25. Número de agrupaciones de artesanos \\
\hline & 26. Número de centros de alumnos \\
\hline & 27. Número de partidos o agrupaciones políticas \\
\hline \multirow[t]{7}{*}{ Dimensión del capital humano } & 28. Número de técnicos y profesionales \\
\hline & 29. Número de postgraduados \\
\hline & 30. Número de alumnos que rinden pruebas de selección universitaria y son aceptados en universidades \\
\hline & 31. Número de profesionales de la comuna que trabajan en otras comunas \\
\hline & 32. Porcentaje de PEA \\
\hline & 33. Número de trabajadores por rama de actividad \\
\hline & 34. Número de pequeñas y medianas empresas \\
\hline \multirow[t]{16}{*}{ Dimensión institucional } & 35. Número de patentes comerciales \\
\hline & 36. Número de ordenanzas municipales \\
\hline & 37. Existencia de Plan de Desarrollo Comunal (PLADECO) vigente \\
\hline & 38. Existencia de Plan Regulador vigente \\
\hline & 39. Existencia de Plan Estratégico Institucional vigente \\
\hline & 40. Existencia de Plan Anual de Salud Municipal (PASAM) vigente \\
\hline & 41. Existencia de Plan Anual de Educación Municipal (PADEM) vigente \\
\hline & 42. Existencia de un sistema de información geográfica (SIG) comunal \\
\hline & 43. Kilómetros de zonas de interés turístico (ZOIT) \\
\hline & 44. Número de servicios financieros \\
\hline & 45. Número servicios de formación \\
\hline & 46. Número de plataformas de salud \\
\hline & 47. Número de plataformas de transporte \\
\hline & 48. Número de plataformas de telecomunicaciones \\
\hline & 49. Número de profesionales por habitante \\
\hline & 50. Número de proyectos ejecutados del FNDR con recomendación técnica favorable \\
\hline
\end{tabular}


Cuadro A1.1 (conclusión)

\begin{tabular}{|c|c|}
\hline \multicolumn{2}{|l|}{ Variables identificadas } \\
\hline \multirow[t]{5}{*}{ Dimensión institucional } & 51. Número de proyectos ejecutados del Fondo Regional de Iniciativa Local (FRIL) \\
\hline & 52. Número de proyectos ejecutados del Programa Mejoramiento Urbano (PMU) \\
\hline & 53. Ingreso autónomo municipal en pesos chilenos \\
\hline & 54. Porcentaje de gastos del presupuesto en salud \\
\hline & 55. Porcentaje de dependencia del Fondo Común Municipal (FCM) \\
\hline \multirow[t]{29}{*}{ Dimensión de infraestructura } & 56. Número de puntos de acceso universal \\
\hline & 57. Número de telecentros \\
\hline & 58. Número de infocentros \\
\hline & 59. Número de bibliotecas asociadas a BiblioRedes \\
\hline & 60. Porcentaje de cobertura de redes viales asfaltadas \\
\hline & 61. Porcentaje de cobertura de redes viales pavimentadas \\
\hline & 62. Número de postas \\
\hline & 63. Número de consultorios \\
\hline & 64. Número de hospitales \\
\hline & 65. Número de camas complejas \\
\hline & 66. Número de sillones dentales \\
\hline & 67. Número de máquinas de rayos $X$ \\
\hline & 68. Número de máquinas de tomografía \\
\hline & 69. Número de escuelas \\
\hline & 70. Número de internados \\
\hline & 71. Número de bibliotecas \\
\hline & 72. Porcentaje de déficit de vivienda \\
\hline & 73.Porcentaje de déficit de materiales de vivienda \\
\hline & 74. Porcentaje de cobertura de agua potable \\
\hline & 75. Porcentaje de cobertura de la red eléctrica \\
\hline & 76. Porcentaje de cobertura de alcantarillado \\
\hline & 77. Número de maquinarias para la producción primaria \\
\hline & 78. Kilómetros de áreas silvestres protegidas por el Estado \\
\hline & 79. Kilómetros de zona marítima \\
\hline & 80. Kilómetros de zona lacustre \\
\hline & 81. Kilómetros de zona fluvial \\
\hline & 82. Presencia de volcanes \\
\hline & 83. Presencia de recursos mineros metálicos \\
\hline & 84. Presencia de recursos no metálicos \\
\hline \multirow[t]{4}{*}{ Dimensión económica } & 85. Inversiones comunales proyectadas en pesos chilenos \\
\hline & 86. Metros cuadrados construidos \\
\hline & 87. Número de permisos de edificación \\
\hline & 88. Exportaciones comunales en pesos chilenos \\
\hline
\end{tabular}

Fuente: Elaboración propia. 


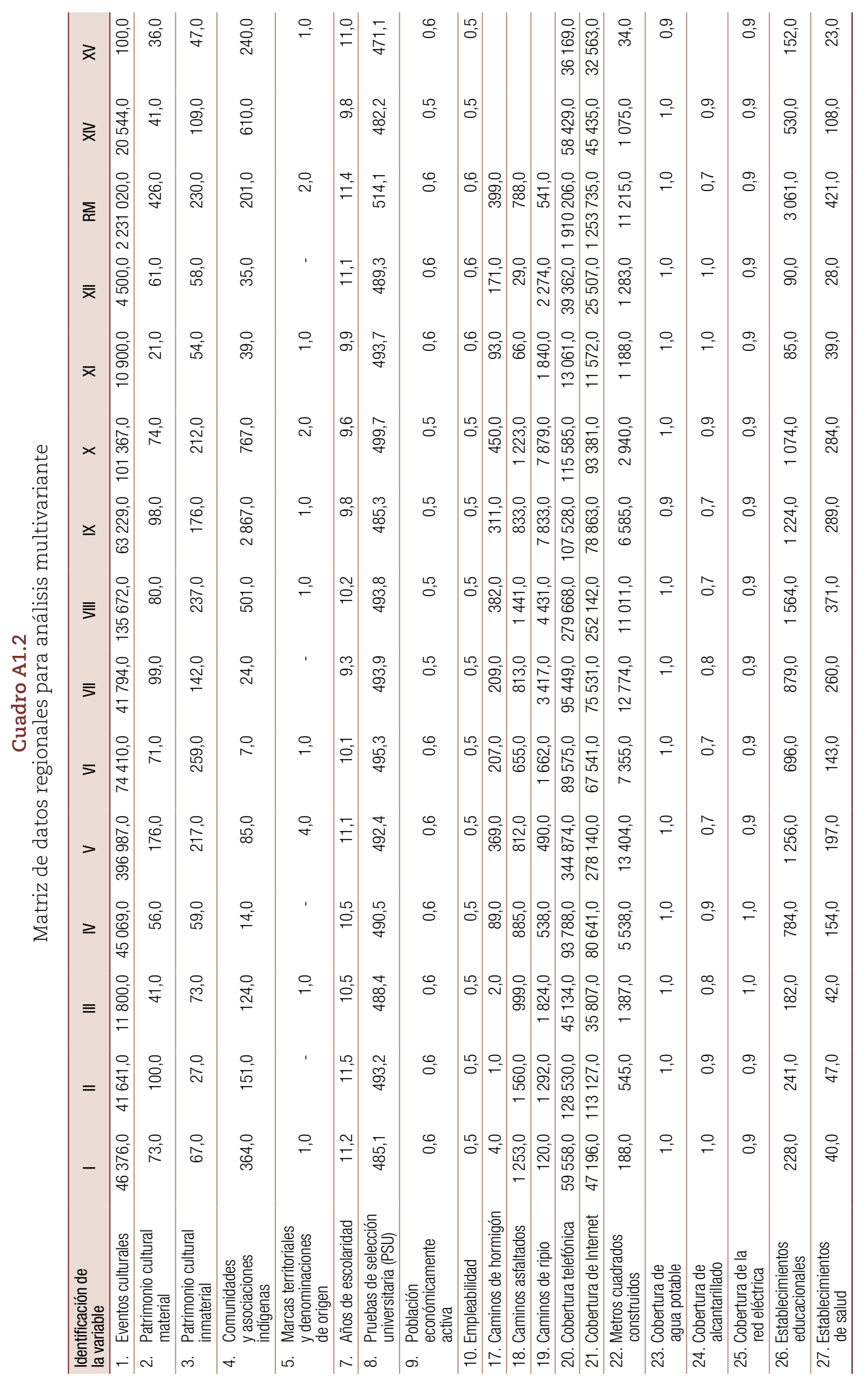




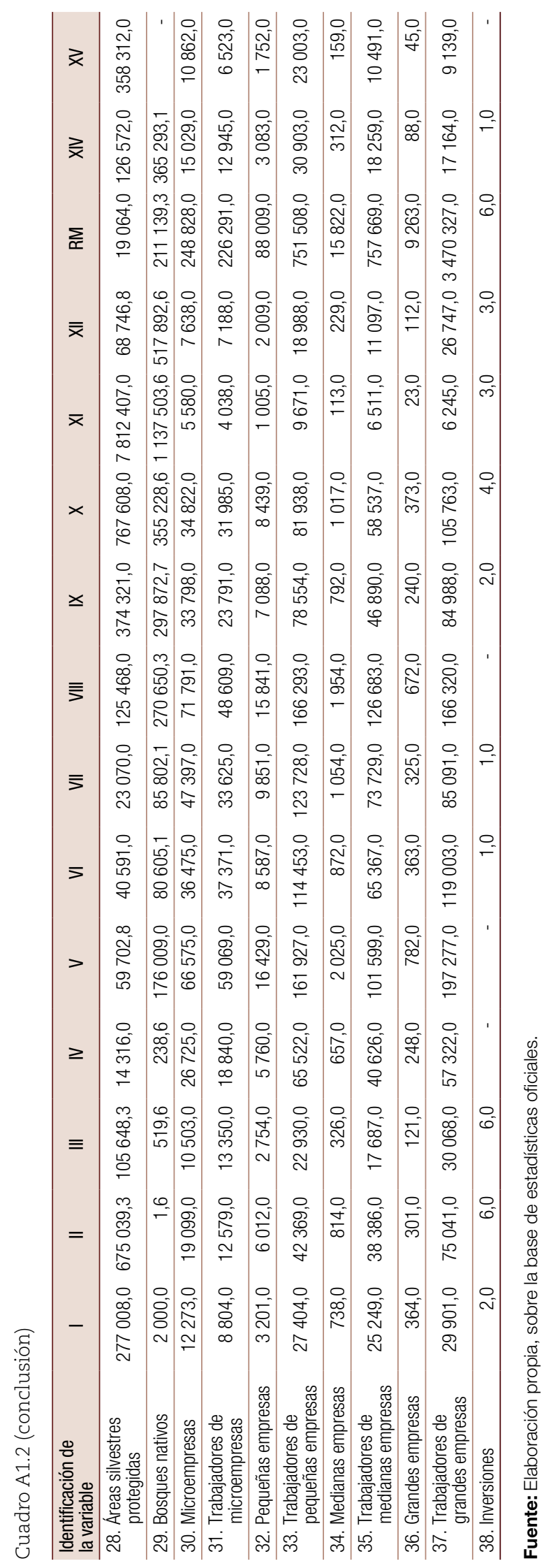

